\title{
Estudios tafonómicos del nivel Auriñaciense Arcaico de la Cueva de El Castillo (Puente Visgo, Cantabria): Los microdesechos líticos
}

\author{
Roberto PeretTI * y Alberto Mingo **
}

\begin{abstract}
RESUMEN ABSTRACT
En este trabajo se analizan los microdesechos líticos del nivel Auriñaciense Arcaico de la Cueva de El Castillo. En el mismo se presenta la metodología y técnica para efectuar este tipo de análisis. Los resultados obtenidos permiten identificar: 1) que se han llevado a cabo actividades de manufactura, uso, modificación y mantenimiento de los utensilios; 2) se han priorizado estrategias tecnológicas conservadoras en detrimento de las

expeditivas; 3) se han localizado posibles áreas de actividad de talla in situ; 4) utilización preferencial de

In this paper we have studied the lithic microdebitage of the Earliest Aurignacian Level of the El Castillo Cave. The methodology and technique to perform this type of analysis also is presented. The results obtained allow to identify: 1) Manufacture, use, modification and maintenance activities of tools that have been implemented; 2) Curated technological strategies have been developed in a bigger way than the expedient ones; 3) Some possible flintworking activity areas have been located; 4) Preferential utilization of
\end{abstract}

* Departamento de Arqueología INCUAPA. UNC. Del Valle 5737. B7400 JWI Olavarría. Argentina. E-mail: rperetti@soc.unicen.edu.ar

** Departamento de Prehistoria e Historia Antigua. Becario predoctoral-UNED. Paseo Senda del Rey 7. 28040 Madrid. España. E-mail: albertomingo@hotmail.com. 
percutores blandos con relación a los duros; 5) selección preferencial de materias primas líticas; 6) finalmente, se han reconocido acumulaciones primarias y secundarias que podrían responder a los procesos postdeposicionales del depósito arqueológico.

PALABRAS CLAVE Microdesechos, sistema de producción lítico, materias primas líticas, atributos tecnológicos, procesos postdeposicionales, conservado, expeditivo, distribuciones espaciales. soft hammers over the hard ones; 5) Raw material selection; 6) finally, it is been recognized primary and secondary clusters that could have had their origin in the postdepositional formation processes of the archaeological deposit.

\section{KEYWORDS}

Microdebitage, lithic production system, raw material, technological features, postdepositional processes, curated, expedient, spatial distributions

\section{INTRODUCCIÓN}

El presente trabajo se enmarca dentro de un proyecto más amplio dirigido por los Drs. Victoria Cabrera Valdés y Federico Bernaldo de Quirós. Éste cuenta con la participación de un equipo multidisciplinario de investigadores. Los estudios desarrollados actualmente tienen por objeto obtener información relevante para contrastar y complejizar los modelos de desarrollo cultural formulados para la Región Cantábrica en la transición del Paleolítico Medio al Superior. Las investigaciones en curso se orientan en torno a cinco ejes principales: 1) análisis faunístico, 2) análisis lítico, 3) análisis paleoambiental, 4) cronología, a través de la obtención e interpretación de nuevas dataciones radiocarbónicas, y 5) estudios tafonómicos y de procesos de formación del registro arqueológico (Bernaldo de Quirós y Cabrera Valdés, 1996; Cabrera Valdés et al., 1993, 1996; Cabrera Valdés y Bernaldo de Quirós, 1996).

El trabajo, que ahora se presenta, aborda específicamente el análisis lítico y no incluye el análisis total del material registrado en el sitio ya que solamente se analizaron los microdesechos provenientes de los niveles $18 \mathrm{c}, 18 \mathrm{c}$ Base y 19 Superior, recuperados en el transcurso de los trabajos de campo realizados durante los años 1986, 1988, 1989 y 1990 en el yacimiento arqueológico Cueva de El Castillo. Los microdesechos recuperados en los demás niveles serán estudiados en una segunda etapa de investigación.

A los fines operativos de este estudio, se consideran como microdesechos líticos a todos los fragmentos menores de $1 \mathrm{~cm}^{2}$ dentro de una amplia 
categoría propuesta por Fish (1981) que incluye a todos los subproductos de preparación del núcleo, etapas de elaboración de útiles y los resultantes de la modificación y mantenimiento durante la vida útil de los artefactos (Fish, 1981:374).

Teniendo en cuenta la asignación de Cueva de El Castillo como sitio de agregación estacional y que la tendencia funcional habría sido de campamento base (Bernaldo de Quirós y Cabrera Valdés, 1996), se espera que estén representados, a través de los microdesechos líticos, algunos eventos producidos en distintas etapas del proceso de producción lítica (Collins, 1975, 1989-1990; Ericson, 1982; Fish, 1981; Nami, 1991a; Patterson y Sollberger, 1978; Shott, 1994; Sullivan y Rozen, 1985). Por lo tanto, el análisis de los microdesechos puede orientar a comprender y explicar las actividades y procesos que conforman el subsistema de producción lítica (Schiffer, 1972).

De este modo, a través del análisis de los microdesechos se intenta identificar, por un lado, aspectos relacionados al aprovechamiento y uso de materias primas líticas y por otro, las etapas del proceso de producción lítica representadas en El Castillo. Especialmente, tomando en cuenta las características de este sitio, se tiene interés en evaluar la variabilidad tecnológica temporal centrando la atención en el registro de los microdesechos correspondientes a los niveles definidos en el sitio.

\section{OBJETIVOS}

Considerando que la tecnología lítica es una vía más de comprensión de los grupos cazadores-recolectores a partir de su relación directa con los condicionamientos materiales, las estrategias económicas y también las estructuras sociales e ideológicas del grupo humano (Bamforth, 1986; Binford, 1978; Torrence, 1989; Shott, 1989 entre otros), este trabajo aspira, no sólo a presentar una contribución concreta a dicho proyecto, para poder comprender mejor la dinámica de formación del depósito arqueológico de éste yacimento, sino también a aportar un caso de estudio que sirva de modelo general en el análisis de microdesechos líticos.

El objetivo general de este trabajo es identificar la serie de actividades relacionadas al subsistema de producción lítico llevadas a cabo en EI Castillo durante la manufactura, el reciclado, el mantenimiento y el uso de instrumentos. Dentro de este objetivo general se incluyen los siguientes objetivos específicos:

Realizar el estudio de los microdesechos líticos, producidos durante la ocupación humana del nivel $18 \mathrm{c}$ en una escala temporal que engloba la 
transición del Paleolítico Medio al Superior, más concretamente el Auriñacience Arcaico.

Analizar y discutir el aprovechamiento de las diferentes materias primas líticas.

Analizar la variabilidad en la distribución espacial (horizontal y vertical) de los microdesechos líticos a nivel intrasitio.

\section{ANTECEDENTES}

\section{Cueva de El Castillo}

Para abordar la problemática planteada se consideró apropiado el estudio de este yacimiento debido a que presenta una escala temporal muy amplia y el registro lítico forma una muestra significativa y adecuada para el análisis de microdesechos.

El Castillo se localiza en el área externa de una amplia red de complejos cársticos que conforman esta cueva, correspondiendo con una amplia sala cortada en su extremo por la evolución de la vertiente dando lugar a una gran cavidad con un vestíbulo de sección pseudo rómbica de 18 a 20 $\mathrm{m}$. de ancho y una altura visible actual alrededor de los $20 \mathrm{~m}$.; se debe destacar que en el pasado estuvo practicamente colmatada por sedimentos (Cabrera Valdés et al., 1993).

El nivel $18 \mathrm{c}$ se caracteriza por arcillas pardas ricas en materia orgánica englobando cantos y bloques de caliza dispersos o en concentraciones irregulares, más abundantes hacia el exterior de la cueva. La estratigrafía es masiva, tendiendo a paralela, con un ligero buzamiento hacia el interior. El análisis de los sedimentos presenta una complejidad puesto que al medio natural se le suman los aportes y transformaciones antrópicas y los efectos de las deformaciones producidas por el desprendimiento de grandes bloques del techo (Cabrera Valdés et al., 1993). Los materiales procedentes del exterior, arcillas con cantos, se han deslizado en masa de forma lenta, suave y plástica por solifluxión, en condiciones climáticas no muy rigurosas (Hoyos, 1979).

Las dataciones por C14 AMS (ca. 40.000 A.P.) obtenidas del nivel 18c, junto con las de L'Arbreda y Abric Romani en Cataluña (ca. 38.000 A.P.), representan las más tempranas apariciones del Auriñaciense de Europa Occidental (Bischoff et al., 1989, 1994; Cabrera Valdés y Bischoff, 1989).

En términos culturales, se considera como un sitio de agregación siendo su tendencia funcional como campamento base (Bernaldo de Quirós y Cabrera Valdés, 1996). 
Los microdesechos líticos en el contexto de las investigaciones arqueológicas

Los análisis de microdesechos líticos cuentan con muy pocos antecedentes, siendo esta línea de investigación escasamente abordada en España y de reciente desarrollo en el resto del mundo (ver Baumler y Downum, 1989; Fladmark, 1982; Hull, 1987; Maíllo Fernándéz, 1998; Patterson y Sollberger, 1978; Peretti, 1997; Shott, 1994 entre otros). No obstante, los estudios referidos al registro arqueológico de caracteristicas microscópicas se vienen desarrollando desde la década de los setenta. Hassan (1978), uno de los pioneros en esta clase de estudios, señaló que los microartefactos a diferencia de los macro están menos expuestos a los procesos de traslado por factores culturales y/o naturales (Hassan, 1978:212).

Los estudios realizados desde esta perspectiva han sido desarrollados por varios investigadores lo que permitió crear distintas vías de análisis entre las que se pueden destacar: identificación de patrones de organización espacial de comportamiento dentro de las estructuras de un sitio, reconstrucción de áreas de actividad e interpretación funcional de sitios, inferencias acerca del carácter y la magnitud de las alteraciones postocupacionales, determinación de técnicas de talla lítica, diferenciación de desechos primarios y secundarios, descubrimiento y detección de sitios en condiciones de baja visibilidad y reconstrucción de los ambientes deposicionales de sitios (Clark, 1986; Dunnell y Stein, 1989; Fladmark, 1982; Hassan, 1978; Hull, 1987; Madsen, 1992; McKellar, 1983; Nielsen, 1994; Nicholson, 1983; Patterson y Sollberger, 1978; Sullivan y Rozen, 1985; Schiffer, 1972).

Un gran número de investigadores comparten el criterio de importancia del estudio de los desechos de talla y enfatizan que estos son un punto importante en la investigación concerniente al Sistema de Producción Lítico y que poseen propiedades particulares generadoras de valiosa información tecnológica (Ericson, 1984; Shott, 1994; Sullivan y Rozen, 1985). Específicamente, los microdesechos se distinguen de otros artefactos al no ser objeto frecuente de transporte sino que una vez depositados permanecen in situ. En consecuencia, el examen de los microdesechos líticos con una dimensión máxima de $1 \mathrm{~cm}^{2}$ puede ser particularmente informativo. Estos desechos que fueron originados durante las etapas de la secuencia de producción lítica son retenidos en las matrices de los sitios arqueológicos y pueden ser analizados desde aspectos funcionales o los producidos por agentes naturales (Clark, 1986; Cowan, 1999; Dunnell y Stein, 1989; Fladmark, 1982; Gallagher, 1977; Hull, 1987; Patterson y Sollberger, 1978; Peretti, 1997).

En este sentido, Morrow (1996) plantea que los artefactos manufacturados en un yacimiento y posteriormente transportados quedaran separados 
de los microdesechos derivados de su manufactura. En esta situación, los microdesechos que quedan en el yacimiento inicial forman un "Ghost" con respecto a los artefactos transportados. Del mismo modo, los útiles que han ingresado a un yacimiento y que no están acompañados de los microdesechos derivados de su manufactura formaran "Orphans". Por otra parte, a medida que el período de ocupación de un yacimiento se incrementa la relativa representación de "Ghost" y "Orphans" dentro del conjunto artefactual debería supuestamente decrecer (Morrow, 1996:357 y 358).

Para tratar de identificar los procesos de formación de sitios se deberían considerar factores tales como la disposición directa, que afecta a los materiales culturales microscópicos en forma diferente que a los materiales macroscópicos. Las distintas etapas de la secuencia de reducción lítica (i.e. manufactura, uso, modificación y mantenimiento opcional) pueden ser identificadas mediante una alta densidad de microdesechos líticos, comparados con las densidades de macrodesechos y los útiles (Hull, 1987:773; Morrow, 1996:358). Por lo tanto, la comparación de objetos pequeños y grandes mediante diagramas de distribución, teniendo en cuenta el tipo de materias primas y las categorias de manufactura de utensilios, permite la identificación de los procesos de formación de sitios específicamente culturales (McKellar, 1983; Morrow, 1996; Schiffer, 1983).

En relación a las distribuciones espaciales, es necesario destacar que la mayoría de los investigadores enfatizan en el desarrollo de este tipo de análisis a nivel intrasitio para esclarecer disposiciones no aleatorias de elementos. Cuando se localizan conjuntos de items en suelos de habitación, éstos suelen ser generalmente interpretados como restos fósiles de actividades humanas (Rick, 1976). Desde el trabajo pionero de Ascher (1961) los investigadores vienen tomando conciencia de la importancia que tienen los estudios orientados hacia este tipo de análisis y los procesos de formación de los depósitos arqueológicos como un medio para comprender mejor la dinámica del comportamiento humano en el pasado (Odell, 1980). Estas investigaciones proporcionan inferencias sobre los procesos responsables, tanto naturales como culturales, de las composiciones de los conjuntos arqueológicos (Deal, 1985; Lange y Rydberg, 1972; Savelle, 1984; Schiffer, 1987; Stevenson, 1991).

\section{CONSIDERACIONES METODOLÓGICAS Y TÉCNICAS}

Desde un punto de vista metodológico, se siguieron los lineamientos analíticos y códigos propuestos por Aschero $(1975,1983)$, Bellelli et al., (1985-1987) y Nami (1991a y b). La identificación de los productos de talla 
se realizó según la presencia de ciertos atributos. En este sentido, se consideró como lascas a aquellos artefactos producidos por la fractura intencional de formas base y en los cuales pueden diferenciarse atributos tecnológicos tales como talón, bulbo, estrías, etc. Asimismo, se distinguió como indiferenciados a aquellos otros artefactos cuyo nivel de fracturación no permite discernir ninguna de las características de las caras dorsal $y$ ventral $y$, por ende, no es posible identificarlos como lascas.

Los microdesechos líticos utilizados en este estudio fueron obtenidos durante las excavaciones de las cuadrículas $\mathrm{J} 14$, J $15, \mathrm{~J} 16, \mathrm{~K} 12, \mathrm{~K} 16$, L 16, M 16, N 15, N 16, N 17 y N 18 y constituyen un total de 6084 piezas. Los niveles 18c, 18c Base y 19 Superior (todos ellos englobados dentro del Auriñaciense Arcaico) serán tomados, como unidades de análisis y comparadas entre sí.

El material proveniente de las excavaciones fue recuperado de distintas formas. Los materiales de planta, por una parte, fueron registrados tridimencionalmente mientras que el material de dimensiones muy pequeñas, por otra parte, fue recogido mediante el uso de cribas de malla fina de $0,04 \mathrm{~mm}$. En una segunda etapa, ya en el laboratorio de la Facultad de Geografía e Historia de la Universidad Nacional de Educación a Distancia (UNED) sita en Madrid, se llevo a cabo la separación de los diferentes materiales contenidos en las bolsas (i.e. óseos, coprolitos, carbones, líticos). Posteriormente, se realizaron los siguientes pasos:

- Análisis macroscópico y microscópico de los microdesechos con objeto de registrar los atributos tecnológicos. Para la observación microscópica de los elementos se usó un video microscopio Olympus (OVM $1000 \mathrm{NM}$ ) de 20 - 50x. Mediante el uso de un calibre manual, se tomaron medidas de largo, ancho y espesor de los microdesechos a fin de determinar un máximo y un mínimo de tamaño en las fracturas. La información recogida fue almacenada en la ficha de análisis propuesta por Bellelli et al., 1985-1987.

- Procesamiento del material a través del programa DELCO (Desechos Líticos Computarizados), metodología ágil que hace posible el análisis del conjunto de atributos controlados (Bellelli et al., 1985-1987). Este análisis, permite identificar y combinar atributos para establecer las etapas de la talla. En este sentido, a manera de ejemplo, se puede citar el caso del tipo de bulbo, el tipo de talón y la presencia y/o ausencia de labio que estarían relacionados con el uso de distintos percutores como los blandos y/o duros (Baumler, 1985; Nami y Bellelli, 1994; Patterson y Sollberger, 1978).

- Utilización del programa EXCEL (versión 7.00) (Microsoft Corporation 1997) en la elaboración de tablas y figuras. 
Las variables y atributos analizados en los microdesechos líticos son los siguientes:

- Materia prima: mediante la observación, macro y microscópica, de la materia prima se puede establecer una escala de aptitud para la talla (Nami y Rabassa, 1988), procedencia, explotación y aprovechamiento diferencial de distintas materias primas, entre otras variables (Nami, $1992 \mathrm{~b}$ ).

- Estado: mediante esta categoría se realiza una primera clasificación de los desechos de talla a partir de la presencia o ausencia de fragmentación (Bellelli et al., 1985-1987). En el mismo pueden intervenir diversos factores como, por ejemplo, la calidad de la materia prima, los accidentes de talla, el pisoteo, el transporte, el uso, etc.

- Tipo: es un indicador tecno-morfológico que permite inferir en que etapa de la secuencia de producción lítica se encuentran los microdesechos (i.e. lascas primarias, de arista, angulares, planas, etc.) (Aschero, 1975, 1983).

- Módulo de Longitud-Anchura y Espesor: consignan dimensiones relativas de largo y ancho utilizando el gráfico de Bagolini (1968), modificado por Aschero (1975, 1983), y una medida absoluta -el espesor- siguiendo el eje de lascado o el morfológico según el caso. Estos atributos se utilizan específicamente en la evaluación de las distintas etapas de manufactura presentes.

- Las características de: talón, bulbo, cono de percusión, estrías y labio, son indicadores de las técnicas de percusión y presión aplicadas con percutores blandos y duros (Baumler, 1985; Crabtree, 1972; Nami 1991a; Nami y Bellelli, 1994; Patterson y Sollberger, 1978).

- Presencia de corteza: esta característica proporciona información sobre el grado de reducción alcanzada en cada etapa de la manufactura (Aschero, 1975 y 1983; Cowan, 1999; Nash, 1996).

Es importante añadir que, con relación a los atributos, la no-existencia de un grado de certeza absoluta acerca de los mismos nos hace considerarlos ausentes.

A partir del uso de estos códigos se ha pretendido establecer variables dependientes y atributos observables en los microdesechos líticos que puedan ser indicadores de cada una de las actividades o etapas que se llevaron a cabo en El Castillo en relación al subsistema tecnológico de confección de instrumentos de líticos (Nami, 1991 a).

Considerando que los productos de la actividad de talla son el resultado de comportamientos humanos, y que estos productos muestran las 
huellas de los procesos que intervinieron o a los que fueron sometidos (i. e. como la técnica aplicada al momento de tallar, que muestra los atributos que son característicos), podemos deducir que el estudio de esos atributos (i. e. talón, bulbo, labio, estrías, ondas y aristas entre otros) puede permitir conocer las actividades que el tallador llevó a cabo (Patterson y Sollberger, 1978:107).

Para identificar los procesos de formación cultural de El Castillo, se implementaron diagramas de distribución sobre la base de los análisis realizados en los microdesechos por nivel, cuadrícula y sector en las áreas excavadas. En cada cuadrícula se analizaron la frecuencia de las distintas materias primas por nivel, con el fin de identificar patrones que pudieron alterar la distribución y el estado de los microdesechos líticos, y así poder evaluar el comportamiento de diferentes matrices con respecto a: migración, dispersión y alteración morfológica de elementos líticos contenidas en ellas.

Para el estudio de los procesos de formación y con el objeto de poder aislar los factores culturales y/o naturales que hayan modificado el depósito arqueológico se consideraron en este trabajo los aportes efectuados por los:

A. Estudios de arqueología experimental (i.e. Gifford-González et al., 1985; Nami, 1991 a y b, 1992 a y b; Nami y Bellelli, 1994; Maíllo Fernández, 1998; Ohnuma y Bergman, 1982; Patterson y Sollberger,1978; Peretti y Curtoni, 1999; entre otros).

B. Estudios de Etnoarqueología (i.e. Binford, 1978; Clark, 1986; Clark et al., 1992; Gallagher, 1977; Guifford y Behrensmeyer, 1977; Gould, 1979; Pokotylo y Hanks, 1989; White y Modjeska, 1978; entre otros).

C. Estudios especificos de micromamíferos (Bocek, 1986 y 1992; Erlandson, 1984; Gómez, 1996; Gómez et al., 1999; entre otros).

D. Acción del pisoteo (Clark, 1986; Clark et al., 1992; Gifford-González et al., 1985; McBrearty et al., 1998; Villa, 1982; entre otros).

E. Acción de las pendientes y del agua (Cahen y Moeyersons, 1977; Glover, 1979; Laville, 1969; Peretti y Curtoni, 1999; Rick, 1976; Schiffer, 1987; entre otros).

En base a los distintos tipos de análisis indicados anteriormente se estudió la distribución vertical y horizontal de los microdesechos líticos dentro de la secuencia de ocupación, evaluando la acción de los distintos agentes culturales y naturales. 


\section{RESULTADOS}

\section{Materias primas líticas}

Dentro de esta variable se han podido identificar en el registro de microdesechos líticos: arenisca, cuarcita, calcita, cuarzo, sílex, ofita, pizarra, y un número de rocas que no han podido ser determinadas y que han sido calificadas como indiferenciadas; también se ha constatado la presencia de restos de caliza, sin embargo su estado de alteración y descomposición no la hace apta de cómputo. Las materias primas identificadas en el nivel 18 del Auriñaciense Arcaico fueron definidas por Cabrera Valdés et al., (1996) y en lo que respecta a la cuarcita y al silex se presentan de la siguiente manera:

Cuarcita: esta aparece en tres variedades:

C1: se presenta en cantos pequeños, de colores variados y de grano muy fino.

C2: de grano fino, de cantos mayores y de color verde.

C3: de grano fino muy compacto con inclusiones de minúsculas puntuaciones de manganeso de color gris opaco.

Silex: esta aparece en dos variedades:

S1: de textura de grano muy fino y homogéneo, de color gris y hueso.

S2: semejante a la anterior y se diferencia por una opacidad muy fuerte.

Respecto del total de microdesechos recuperados en el sitio $(N=6084)$ se aprecia a la cuarcita 1 (C1), con un porcentaje del 74,69\%, como la más representada. A ésta le siguen en cantidad sílex 1 (S1) con 11,82\%, cuarzo con $4,39 \%$, cuarcita 2 (C2) con $3,96 \%$, sílex 2 (S2) con $3,04 \%$, indiferenciadas con $1,50 \%$ y finalmente arenisca, cuarcita 3 (C3), ofita y pizarra no superan el $0,5 \%$ (tabla 1 ).

Observando los porcentajes de las materias primas en cada nivel (18 c, 18 c Base y 19 Superior) respecto del total (tabla 1), podemos apreciar que existen pequeñas diferencias, aunque la tendencia es la misma (Tabla 2). En la $C 1$, que es la que predomina, al $18 \mathrm{c}$ le corresponde el $53,17 \%$, al 18 c Base el $3,39 \%$ y al 19 Superior el $18,13 \%$. El S1 presenta una porcentaje de $8,88 \%$ en el $18 \mathrm{c}, 0,58 \%$ en el $18 \mathrm{c}$ Base, y $2,37 \%$ en el 19 Superior. El resto de materias primas se comportan en los tres niveles de manera semejante. 
Estudios tafonómicos del nivel Auriñaciense Arcaico de la Cueva de El Castillo...

Tabla 1. Materias primas liticas de la muestra general

\begin{tabular}{lrr}
\hline & $N$ & $\%$ \\
\hline Arenisca & 7 & 0,12 \\
Cuarcita 1 & 4544 & 74,69 \\
Cuarcita 2 & 241 & 3,96 \\
Cuarcita 3 & 3 & 0,05 \\
Calcita & 1 & 0,02 \\
Cuarzo & 267 & 4,39 \\
Indiferenciada & 91 & 1,50 \\
Ofita & 24 & 0,39 \\
Pizarra & 2 & 0,03 \\
Silex 1 & 719 & 11,82 \\
Silex 2 & 185 & 3,04 \\
Muestra General & 6084 & 100,00 \\
\hline
\end{tabular}

Tabla 2. Materias primas líticas de la muestra general por niveles

\begin{tabular}{lrrrrrrrrr}
\hline Nivel & \multicolumn{2}{c}{$18 c$} & \multicolumn{2}{c}{$18 c$ Base } & \multicolumn{2}{c}{19 Superior } & \multicolumn{2}{c}{ Total } \\
\hline Materia prima & \multicolumn{1}{c}{$N$} & \multicolumn{1}{c}{$\%$} & \multicolumn{1}{c}{$N$} & $\%$ & $N$ & $\%$ & $N$ & $\%$ \\
\hline Arenisca & 5 & 0,08 & 1 & 0,02 & 1 & 0,02 & 7 & 0,12 \\
Cuarcita 1 & 3235 & 53,17 & 206 & 3,39 & 1103 & 18,13 & 4544 & 74,69 \\
Cuarcita 2 & 206 & 3,39 & 9 & 0,15 & 26 & 0,43 & 241 & 3,96 \\
Cuarcita 3 & 3 & 0,05 & - & - & - & - & 3 & 0,05 \\
Calcita & 1 & 0,02 & - & - & - & - & 1 & 0,02 \\
Cuarzo & 207 & 3,40 & 13 & 0,21 & 47 & 0,77 & 267 & 4,39 \\
Indiferenciadas & 91 & 1,50 & - & - & - & - & 91 & 1,50 \\
Ofita & 21 & 0,35 & 2 & 0,03 & 1 & 0,02 & 24 & 0,39 \\
Pizarra & 1 & 0,02 & 1 & 0,02 & - & - & 2 & 0,03 \\
Silex 1 & 540 & 8,88 & 35 & 0,58 & 144 & 2,37 & 719 & 11,82 \\
Sílex 2 & 106 & 1,74 & 20 & 0,33 & 59 & 0,97 & 185 & 3,04 \\
\hline Muestra general & 4416 & 72,58 & 287 & 4,72 & 1381 & 22,70 & 6084 & 100,00 \\
\hline
\end{tabular}

\section{Estado de Fragmentación}

Considerando la totalidad de los microdesechos $(\mathrm{N}=6084)$ (Tabla 3 ), se observa a las lascas indiferenciadas como las más representadas $(48,37 \%)$, seguidas por las lascas enteras $(23,80 \%)$ y las lascas fracturadas $\sin$ talón $(17,29 \%)$. Cabe destacar que, las lascas fracturadas con talón son las menos representadas con un 10,54\%. Los niveles, de igual modo, se comportan de la misma manera presentando una tendencia similar puesto que en todos se constata un claro predomino de las lascas 
indiferenciadas (46,58\% para el $18 \mathrm{c}, 53,31 \%$ para el $18 \mathrm{c}$ Base y $53,08 \%$ para el 19 Superior). A pesar de esta aparente uniformidad se aprecian algunas irregularidades en las proporciones de los porcentajes que modifican en parte el comportamiento observado en la muestra general, no obstante, éstas no son en ningún caso suficientemente importantes. Las mismas son: el incremento de las lascas fracturadas con talón en el nivel $18 \mathrm{c}$ Base respecto de las lascas fracturadas sin talón en el mismo nivel, y un descenso significativo de las lascas fracturadas con talón en el nivel 19 Superior (Tabla 3).

Tabla 3. Estado de los microdesechos por nivel

\begin{tabular}{lrrrrrrrrrr}
\hline Estado & Entera & \multicolumn{2}{c}{$\begin{array}{c}\text { Fracturada } \\
\text { con talón }\end{array}$} & \multicolumn{2}{c}{$\begin{array}{c}\text { Fracturada } \\
\text { sin talón }\end{array}$} & \multicolumn{2}{c}{ Indiferenciada } & \multicolumn{2}{c}{ Totales } \\
\hline Nivel & $N$ & $\%$ & \multicolumn{1}{c}{$N$} & $\%$ & $N$ & $\%$ & $N$ & $\%$ & $N$ & $\%$ \\
\hline $18 \mathrm{c}$ & 1045 & 23,66 & 502 & 11,37 & 812 & 18,39 & 2057 & 46,58 & 4416 & 100,00 \\
$18 \mathrm{c}$ Base & 55 & 19,16 & 45 & 15,68 & 34 & 11,85 & 153 & 53,31 & 287 & 100,00 \\
19 Superior & 348 & 25,20 & 94 & 6,81 & 206 & 14,92 & 733 & 53,08 & 1381 & 100,00 \\
\hline Muestra general & 1448 & 23,80 & 641 & 10,54 & 1052 & 17,29 & 2943 & 48,37 & 6084 & 100,00 \\
\hline
\end{tabular}

El análisis del estado de fragmentación por materia prima (figura 1) nos proporciona una valiosa información. La misma consiste en que la frecuencia de lascas enteras es muy alta en el sílex (sobre todo S2) y en $\mathrm{C} 2$ a diferencia de $\mathrm{C} 1$ y cuarzo (CZC).

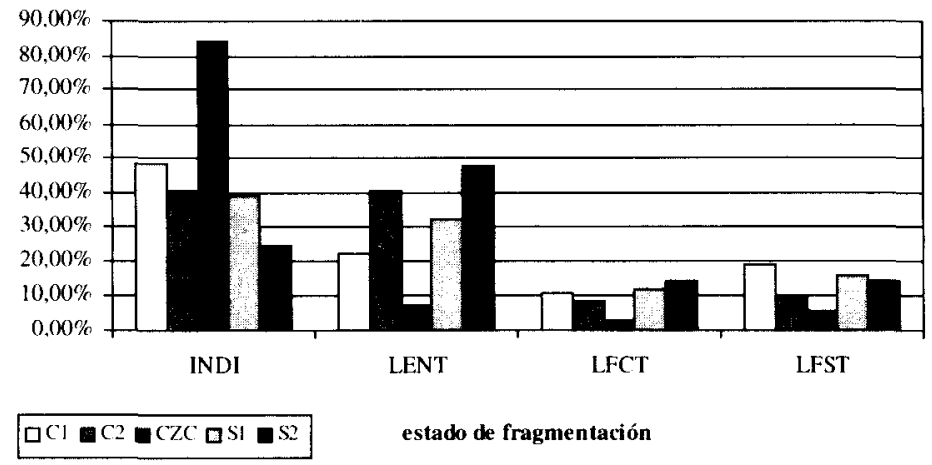

Figura 1. Estado por materia prima. Referencias: C1: cuarcita 1, C2: Cuarcita 2, CZC: cuarzo, S1: silex 1, S2: silex 2, INDI: indiferenciada, LENT: lasca entera, LFCT: lasca fracturada con talón, LFST: lasca fracturada sin talón. 


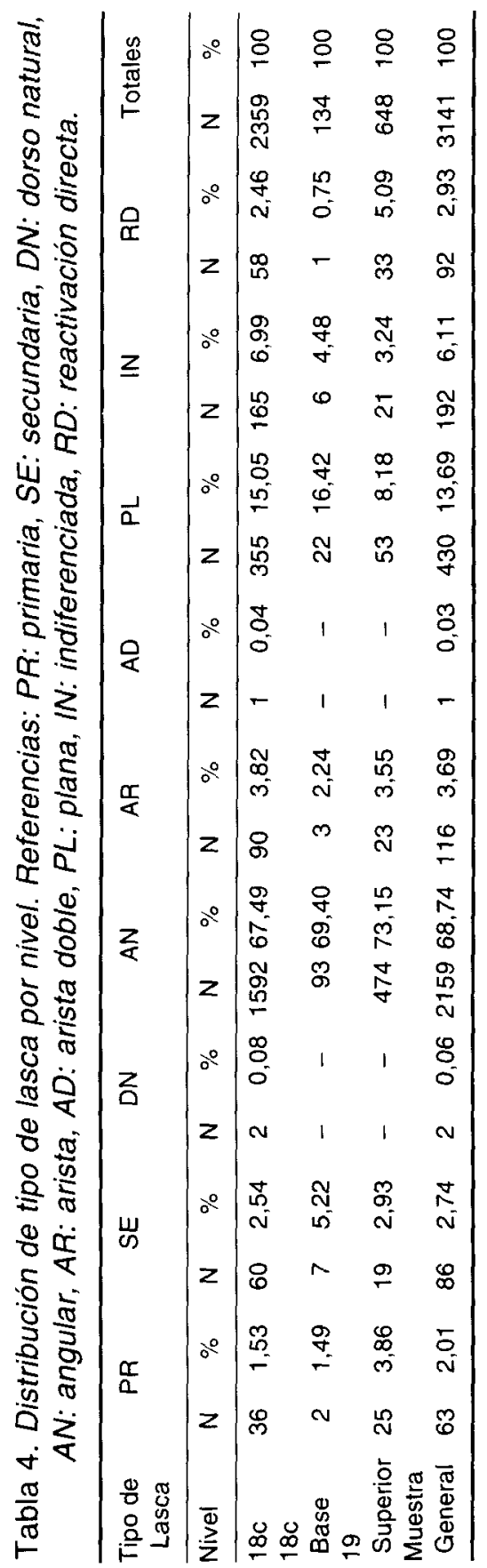

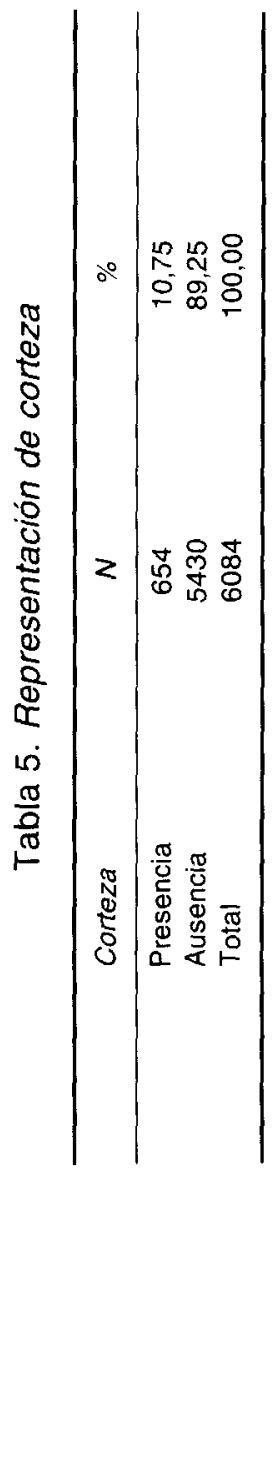




\section{Tipo de lasca}

Se han analizado 3141 elementos del total no habiéndose considerado los indiferenciados (tabla 4). De estos resultados se puede observar que las lascas angulares dominan ampliamente el conjunto con un $68,74 \%$, seguidas por las planas con un $13,69 \%$ y las indiferenciadas con un $6,11 \%$ (a este segmento corresponden las lascas que aún presentando atributos claros no se las puede englobar en otras categorías porque presentan fracturas, de modo que no se puede reconocer de una forma segura el tipo de lasca). El resto de las categorías, no supera el $3 \%$, mientras que las de dorso natural y arista doble presentan una frecuencia casi nula. El registro por niveles se comporta de manera similar al conjunto, en donde las angulares mantienen una amplia representatividad. No obstante, se notan pequeñas variaciones porcentuales que no modifican sustancialmente la interpretación de estos resultados. Por su parte, la presencia de corteza junto a las lascas primarias y secundarias representan con respecto al resto del conjunto el 10,75\% (tabla 5). En este sentido, la materia prima que presenta mayor frecuencia de corteza (tabla 6 ) es la cuarcita (C1) con un $92,05 \%$ y el resto no supera el $3 \%$.

Tabla 6. Materia prima que presenta reserva de corteza

\begin{tabular}{lcc}
\hline Materia Prima & $N$ & $\%$ \\
\hline Arenisca & & \\
Cuarcita 1 & 602 & 92,05 \\
Cuarcita 2 & 12 & 1,83 \\
Cuarcita 3 & - & - \\
Calcita & - & - \\
Cuarzo 9 & 1,38 & \\
Indiferenciada & 2 & 0,31 \\
Ofita & 4 & 0,61 \\
Pizarra & - & - \\
Sílex 1 & 15 & 2,29 \\
Sílex 2 & 10 & 1,53 \\
\hline Muestra General & 654 & 100,00 \\
\hline
\end{tabular}

Módulo longitud anchura

El análisis de este atributo (tabla 7) para un total de 1448 microdesechos enteros (considerando lo expuesto por Bellelli et al.,1985-1987) en que deben realizarse sobre lascas enteras), se observa un predominio importante de los mediano normal $(38,47 \%)$, le siguen el corto ancho $(23,07 \%)$, el mediano alargado 
$(16,16 \%)$, corto muy ancho $(12,98 \%)$ y el corto anchísimo $(7,67 \%)$, finalmente

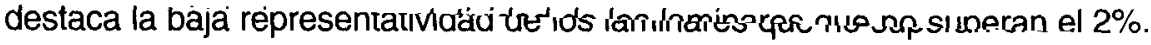

Para complementar esta información se presenta la escala de intervalos de los espesores registrados en la totalidad de la muestra (figura 2) en donde se observa la predominancia de los más delgados sobre el resto.

Tabla 7. Módulo de longitud anchura de los microdesechos enteros por nivel

\begin{tabular}{|c|c|c|c|c|c|c|c|c|}
\hline & \multicolumn{2}{|c|}{$18 \mathrm{c}$} & \multicolumn{2}{|c|}{ 18c Base } & \multicolumn{2}{|c|}{19 Superior } & \multicolumn{2}{|c|}{ Totales } \\
\hline & $N$ & $\%$ & $N$ & $\%$ & $N$ & $\%$ & $N$ & $\%$ \\
\hline Laminar muy angosto & 1 & 0,10 & - & - & - & - & 1 & 0,07 \\
\hline Laminar angosto & 2 & 0,19 & - & - & - & - & 2 & 0,14 \\
\hline Laminar normal & 21 & 2,01 & - & - & - & - & 21 & 1,45 \\
\hline Mediano alargado & 186 & 17,80 & 10 & 18,18 & 38 & 10,92 & 234 & 16,16 \\
\hline Mediano normal & 405 & 38,76 & 22 & 40,00 & 130 & 37,36 & 557 & 38,47 \\
\hline Corto & 245 & 23,44 & 16 & 29,09 & 73 & 20,98 & 334 & 23,07 \\
\hline Corto muy ancho & 128 & 12,25 & 4 & 7,27 & 56 & 16,09 & 188 & 12,98 \\
\hline Corto anchisimo & 57 & 5,45 & 3 & 5,45 & 51 & 14,66 & 111 & 7,67 \\
\hline Muestra general & 1045 & 100,00 & 55 & 100,00 & 348 & 100,00 & 1448 & 100,00 \\
\hline
\end{tabular}

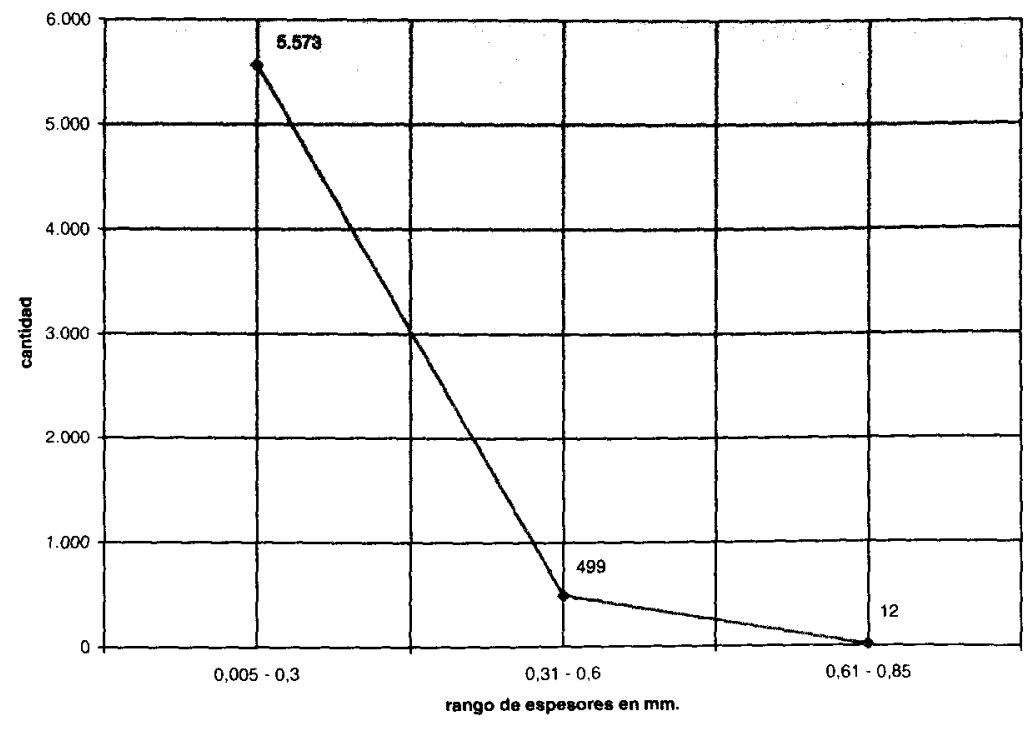

Figura 2. Distribución de espesores. 


\section{Talones y bulbos}

Tomando en consideración el total de microdesechos $(\mathrm{N}=6084)$ se han podido identificar en los mismos 2089 tipo de talones que se distribuyen entre lascas enteras y fracturadas con talón (tabla 8). Como se puede observar, los talones preparados son los más frecuentes y el más representado es el liso $(51,84 \%)$, seguido por el filiforme $(22,45 \%)$, el facetado $(8,23 \%)$, el puntiforme $(6,94 \%)$, el indiferenciado $(2,78 \%)$ y el diedro $(2,54 \%)$. Para los talones no preparados (corticales) se puede ver que presentan una baja frecuencia $(5,22 \%)$ con respecto al total.

En cuanto al bulbo, considerando las lascas enteras y las fracturadas con y sin talón que presentaban este atributo (tabla 9) pudo observarse un total de 2676 tipo de bulbos, entre los cuales el más frecuente es el difuso $(60,16 \%)$ seguido por el pronunciado $(28,66 \%)$ y el indiferenciado $(11,17 \%)$.

Tabla 8. Frecuencia de talones

\begin{tabular}{lrr}
\hline Tipo de talones & $N$ & \multicolumn{1}{c}{$\%$} \\
\hline Cortical & 109 & $5,22 \%$ \\
Diedro & 53 & $2,54 \%$ \\
Facetado & 172 & $8,23 \%$ \\
Filiforme & 469 & $22,45 \%$ \\
Indiferenciado & 58 & $2,78 \%$ \\
Liso & 1083 & $51,84 \%$ \\
Puntiforme & 145 & $6,94 \%$ \\
\hline Totales & 2089 & $100,00 \%$ \\
\hline
\end{tabular}

Tabla 9. Frecuencia de bulbos

\begin{tabular}{lrc}
\hline Tipo de bulbo & $N$ & $\%$ \\
\hline Difuso & 1610 & 60,16 \\
Indiferenciado & 299 & 11,17 \\
Pronunciado & 767 & 28,66 \\
\hline Total & 2676 & 100,00 \\
\hline
\end{tabular}

Para esclarecer un poco más estos resultados se decidió realizar un análisis más detallado de estos dos atributos combinando los mismos por nivel y materias primas (tablas 10,11, 12 y 13). No fueron consideradas en este análisis por su bajo número (ya que sobredimensionarían los re- 
Estudios tafonómicos del nivel Auriñaciense Arcaico de la Cueva de El Castillo...

sultados) las siguientes materias primas: arenisca, calcita, cuarcita (C3), indeferenciadas, pizarra, ofita y cuarzo.

Tabla 10. Cantidades y porcentajes de talones y bulbos por niveles en $C 1$

\begin{tabular}{|c|c|c|c|c|c|c|c|c|c|}
\hline \multirow[b]{2}{*}{ Nivel } & \multirow{2}{*}{$\begin{array}{l}\text { Tipo de bulbo } \\
\text { Tipo de talón }\end{array}$} & \multicolumn{2}{|c|}{ Difuso } & \multicolumn{2}{|c|}{ Indiferenciado } & \multicolumn{2}{|c|}{ Pronunciado } & \multicolumn{2}{|c|}{ Total general } \\
\hline & & $N$ & $\%$ & $N$ & $\%$ & $N$ & $\%$ & $N$ & $\%$ \\
\hline \multirow[t]{7}{*}{$18 \mathrm{c}$} & Cortical & 40 & 2,68 & 15 & 1,00 & 25 & 1,67 & 80 & 5,35 \\
\hline & Diedro & 9 & 0,60 & 2 & 0,13 & 14 & 0,94 & 25 & 1,67 \\
\hline & Facetado & 43 & 2,88 & 6 & 0,40 & 21 & 1,41 & 70 & 4,69 \\
\hline & Filiforme & 161 & 10,78 & 24 & 1,61 & 48 & 3,21 & 233 & 15,60 \\
\hline & Indiferenciado & 21 & 1,41 & 9 & 0,60 & 15 & 1,00 & 45 & 3,01 \\
\hline & Liso & 322 & 21,55 & 84 & 5,62 & 169 & 11,31 & 575 & 38,49 \\
\hline & Puntiforme & 39 & 2,61 & 7 & 0,47 & 16 & 1,07 & 62 & 4,15 \\
\hline Total & $18 \mathrm{c}$ & 635 & 42,50 & 147 & 9,84 & 308 & 20,62 & 1090 & 72,96 \\
\hline \multirow[t]{7}{*}{ 18c Base } & Cortica & 1 & 0,07 & 2 & 0,13 & 1 & 0,07 & 4 & 0,27 \\
\hline & Diedro & 1 & 0,07 & 1 & 0,07 & 1 & 0,07 & 3 & 0,20 \\
\hline & Facetado & 1 & 0,07 & - & - & 1 & 0,07 & 2 & 0,13 \\
\hline & Filiforme & 15 & 1,00 & 3 & 0,20 & 4 & 0,27 & 22 & 1,47 \\
\hline & Indiferenciado & 1 & 0,07 & - & - & - & - & 1 & 0,07 \\
\hline & Liso & 17 & 1,14 & 9 & 0,60 & 13 & 0,87 & 39 & 2,61 \\
\hline & Puntiforme & 4 & 0,27 & 1 & 0,07 & - & - & 5 & 0,33 \\
\hline Total & 18c Base & 40 & 2,68 & 16 & 1,07 & 20 & 1,34 & 76 & 5,09 \\
\hline \multirow[t]{7}{*}{19 Superior } & Cortical & 13 & 0,87 & 1 & 0,07 & 4 & 0,27 & 18 & 1,20 \\
\hline & Diedro & 1 & 0,07 & - & - & 1 & 0,07 & 2 & 0,13 \\
\hline & Facetado & 20 & 1,34 & 4 & 0,27 & 11 & 0,74 & 35 & 2,34 \\
\hline & Filiforme & 69 & 4,62 & 9 & 0,60 & 14 & 0,94 & 92 & 6,16 \\
\hline & Indiferenciado & 1 & 0,07 & - & - & - & - & $\uparrow$ & 0,07 \\
\hline & Liso & 116 & 7,76 & 14 & 0,94 & 33 & 2,21 & 163 & 10,91 \\
\hline & Puntiforme & 7 & 0,47 & 2 & 0,13 & 8 & 0,54 & 17 & 1,14 \\
\hline \multicolumn{2}{|c|}{ Total 19 Superior } & 227 & 15,19 & 30 & 2,01 & 71 & 4,75 & 328 & 21,95 \\
\hline \multicolumn{2}{|l|}{ Muestra } & 902 & 60,37 & 193 & 12,92 & 399 & 26,71 & 1494 & 100,00 \\
\hline
\end{tabular}

Tabla 11. Cantidades y porcentajes de talones y bulbos por niveles en C2

\begin{tabular}{llccccccccc}
\hline & Tipo de bulbo & \multicolumn{2}{c}{ Difuso } & \multicolumn{3}{c}{ Indiferenciado } & Pronunciado & \multicolumn{2}{c}{ Totales } \\
\hline Nivel & Tipo de talón & N & $\%$ & $N$ & $\%$ & $N$ & $\%$ & $N$ & $\%$ \\
\hline \multirow{2}{*}{$18 \mathrm{c}$} & Cortical & 1 & 0,85 & - & - & - & - & 1 & 0,85 \\
& Diedro & - & - & 2 & 1,69 & 2 & 1,69 & 4 & 3,39 \\
& Facetado & 6 & 5,08 & 1 & 0,85 & 3 & 2,54 & 10 & 8,47 \\
\hline
\end{tabular}


Tabla 11. (Continuación)

\begin{tabular}{llrrrrrrrrr}
\hline & Tipo de bulbo & \multicolumn{2}{c}{ Difuso } & \multicolumn{2}{c}{ Indiferenciado } & \multicolumn{2}{c}{ Pronunciado } & \multicolumn{2}{c}{ Totales } \\
\hline Nivel & Tipo de talón & \multicolumn{1}{c}{$N$} & $\%$ & $N$ & $\%$ & $N$ & $\%$ & $N$ & $\%$ \\
\hline & Filiforme & 7 & 5,93 & 2 & 1,69 & 2 & 1,69 & 11 & 9,32 \\
& Indiferenciado & 1 & 0,85 & - & - & - & - & 1 & 0,85 \\
& Liso & 46 & 38,98 & 17 & 14,41 & 10 & 8,47 & 73 & 61,86 \\
& Puntiforme & 2 & 1,69 & 1 & 0,85 & - & - & 3 & 2,54 \\
\hline Total & 18c & 63 & 53,39 & 23 & 19,49 & 17 & 14,41 & 103 & 87,29 \\
\hline 18c Base & Filiforme & 1 & 0,85 & - & - & - & - & 1 & 0,85 \\
& Liso & 1 & 0,85 & 1 & 0,85 & 1 & 0,85 & 3 & 2,54 \\
\hline Total 18c Base & 2 & 1,69 & 1 & 0,85 & 1 & 0,85 & 4 & 3,39 \\
\hline 19 Superior & Facetado & 1 & 0,85 & - & - & - & - & 1 & 0,85 \\
& Filiforme & - & - & 1 & 0,85 & - & - & 1 & 0,85 \\
& Liso & 6 & 5,08 & 1 & 0,85 & 2 & 1,69 & 9 & 7,63 \\
\hline \multicolumn{1}{l}{ Total 19 Superior } & 7 & 5,93 & 2 & 1,69 & 2 & 1,69 & 11 & 9,32 \\
\hline \multicolumn{2}{l}{ Muestra general } & 72 & 61,02 & 26 & 22,03 & 20 & 16,95 & 118 & 100,00 \\
\hline
\end{tabular}

Tabla 12. Cantidades y porcentajes de talones y bulbos por niveles en $S 1$

\begin{tabular}{llrrrrrrrr}
\hline & Tipo de bulbo & \multicolumn{2}{c}{ Difuso } & \multicolumn{3}{c}{ Indiferenciado } & Pronunciado & \multicolumn{2}{c}{ Totales } \\
\hline Nivel & Tipo de talón & \multicolumn{1}{c}{$N$} & \multicolumn{1}{c}{$\%$} & $N$ & $\%$ & $N$ & $\%$ & $N$ & $\%$ \\
\hline 18c & Diedro & 3 & 0,94 & - & - & 7 & 2,19 & 10 & 3,13 \\
& Facetado & 13 & 4,06 & 3 & 0,94 & 14 & 4,38 & 30 & 9,38 \\
& Filiforme & 28 & 8,75 & 8 & 2,50 & 15 & 4,69 & 51 & 15,94 \\
& Indiferenciado & 6 & 1,88 & - & - & 1 & 0,31 & 7 & 2,19 \\
& Liso & 58 & 18,13 & 19 & 5,94 & 36 & 11,25 & 113 & 35,31 \\
& Puntiforme & 22 & 6,88 & 6 & 1,88 & 4 & 1,25 & 32 & 10,00 \\
\hline Total & 18c & 130 & 40,63 & 36 & 11,25 & 77 & 24,06 & 243 & 75,94 \\
\hline 18c Base & Diedro & 1 & 0,31 & 1 & 0,31 & 1 & 0,31 & 3 & 0,94 \\
& Facetado & 1 & 0,31 & - & - & - & - & 1 & 0,31 \\
& Indiferenciado & 1 & 0,31 & - & - & 1 & 0,31 & 2 & 0,63 \\
& Liso & 2 & 0,63 & 1 & 0,31 & 2 & 0,63 & 5 & 1,56 \\
& Puntiforme & 2 & 0,63 & - & - & 1 & 0,31 & 3 & 0,94 \\
\multirow{2}{*}{ Total 18c Base } & 7 & 2,19 & 2 & 0,63 & 5 & 1,56 & 14 & 4,38 \\
19 Superior & Facetado & 6 & 1,88 & 1 & 0,31 & 2 & 0,63 & 9 & 2,81 \\
& Filiforme & 15 & 4,69 & 1 & 0,31 & 4 & 1,25 & 20 & 6,25 \\
& Indiferenciado & - & - & - & - & 1 & 0,31 & 1 & 0,31 \\
& Liso & 11 & 3,44 & 4 & 1,25 & 9 & 2,81 & 24 & 7,50 \\
& Puntiforme & 5 & 1,56 & 2 & 0,63 & 2 & 0,63 & 9 & 2,81 \\
\hline Total 19 Superior & 37 & 11,56 & 8 & 2,50 & 18 & 5,63 & 63 & 19,69 \\
\hline Muestra general & 174 & 54,38 & 46 & 14,38 & 100 & 31,25 & 320 & 100,00 \\
\hline
\end{tabular}


Estudios tafonómicos del nivel Auriñaciense Arcaico de la Cueva de El Castillo...

Tabla 13. Cantidades y porcentajes de talones y bulbos por niveles en $S 2$

\begin{tabular}{|c|c|c|c|c|c|c|c|c|c|}
\hline \multirow[b]{2}{*}{ Nivel } & \multirow{2}{*}{$\frac{\text { Tipo de bulbo }}{\text { Tipo de talón }}$} & \multicolumn{2}{|c|}{ Difuso } & \multicolumn{2}{|c|}{ Indiferenciado } & \multicolumn{2}{|c|}{ Pronunciado } & \multicolumn{2}{|c|}{ Totales } \\
\hline & & $N$ & $\%$ & $N$ & $\%$ & $N$ & $\%$ & $N$ & $\%$ \\
\hline \multirow[t]{6}{*}{$18 c$} & Cortical & - & - & 1 & 0,88 & - & - & 1 & 0,88 \\
\hline & Diedro & 2 & 1,75 & - & - & 1 & 0,88 & 3 & 2,63 \\
\hline & Facetado & 3 & 2,63 & - & - & 3 & 2,63 & 6 & 5,26 \\
\hline & Filiforme & 7 & 6,14 & 3 & 2,63 & 6 & 5,26 & 16 & 14,04 \\
\hline & Liso & 17 & 14,91 & 6 & 5,26 & 12 & 10,53 & 35 & 30,70 \\
\hline & Puntiforme & 4 & 3,51 & 3 & 2,63 & 4 & 3,51 & 11 & 9,65 \\
\hline \multicolumn{2}{|l|}{ Total $18 \mathrm{c}$} & 33 & 28,95 & 13 & 11,40 & 26 & 22,81 & 72 & 63,16 \\
\hline \multirow[t]{3}{*}{ 18c Base } & Diedro & - & - & - & - & 1 & 0,88 & 1 & 0,88 \\
\hline & Filiforme & 1 & 0,88 & - & - & - & - & 1 & 0,88 \\
\hline & Liso & 2 & 1,75 & 1 & 0,88 & 1 & 0,88 & 4 & 3,51 \\
\hline \multicolumn{2}{|c|}{ Total 18c Base } & 3 & 2,63 & 1 & 0,88 & 2 & 1,75 & 6 & 5,26 \\
\hline \multirow[t]{6}{*}{19 Superior } & Cortical & 1 & 0,88 & - & - & - & - & 1 & 0,88 \\
\hline & Diedro & 1 & 0,88 & - & - & - & - & 1 & 0,88 \\
\hline & Facetado & 3 & 2,63 & 1 & 0,88 & 1 & 0,88 & 5 & 4,39 \\
\hline & Filiforme & 11 & 9,65 & 1 & 0,88 & 2 & 1,75 & 14 & 12,28 \\
\hline & Liso & 12 & 10,53 & 1 & 0,88 & 1 & 0,88 & 14 & 12,28 \\
\hline & Puntiforme & 1 & 0,88 & - & - & - & - & 1 & 0,88 \\
\hline \multicolumn{2}{|c|}{ Total 19 Superior } & 29 & 25,44 & 3 & 2,63 & 4 & 3,51 & 36 & 31,58 \\
\hline \multicolumn{2}{|c|}{ Muestra general } & 65 & 57,02 & 17 & 14,91 & 32 & 28,07 & 114 & 100,00 \\
\hline
\end{tabular}

\section{Lascas de reactivación}

Del total de microdesechos ( $\mathrm{N}=6084$ ) se pudo distinguir claramente 92 piezas de este tipo de lasca en donde 58 de las mismas, aparecen en el nivel $18 \mathrm{c}, 33$ en el 19 Superior y solo 1 en el $18 \mathrm{c}$ Base (tablas 14 y 15). En lo relativo a la materia prima, la cuarcita (C1) es la más representada ( $75,86 \%$ para el 18 c y $78,79 \%$ para el 19 Superior), le siguen el S2 (8,62\% para el 18 c y $12,12 \%$ para el 19 Superior), el $\mathrm{S} 1(5,17 \%$ en el 18 c y $9,09 \%$ en el 19 Superior), y por último el C2 y la Ofita (con $8,62 \%$ y $1,72 \%$ respectivamente, para el nivel $18 \mathrm{c}$, estando ausentes en el 19 Superior). 
Tabla 14. Nivel $18 \mathrm{c}$

\begin{tabular}{lrrrrrrrrrrrrr}
\hline Materia prima & \multicolumn{2}{c}{ Cuarcita 1 } & \multicolumn{2}{c}{ Cuarcita 2 } & \multicolumn{2}{c}{ Ofita } & \multicolumn{2}{c}{ Silex 1 } & \multicolumn{2}{c}{ Silex 2 } & \multicolumn{2}{c}{ Totales } \\
\hline Cuadricula & $N$ & $\%$ & $N$ & $\%$ & $N$ & $\%$ & $N$ & $\%$ & $N$ & $\%$ & $N$ & $\%$ \\
\hline K16 & 7 & 12,07 & 1 & 1,72 & - & - & 3 & 5,17 & 1 & 1,72 & 12 & 20,69 \\
L16 & 1 & 1,72 & - & - & - & - & - & - & - & - & 1 & 1,72 \\
M16 & 1 & 1,72 & - & - & - & - & - & - & - & - & 1 & 1,72 \\
N16 & 9 & 15,52 & 2 & 3,45 & 1 & 1,72 & - & - & 2 & 3,45 & 14 & 24,14 \\
N17 & 14 & 24,14 & - & - & - & - & - & - & 2 & 3,45 & 16 & 27,59 \\
N18 & 12 & 20,69 & 2 & 3,45 & - & - & - & - & - & - & 14 & 24,14 \\
\hline Muestra general & 44 & 75,86 & 5 & 8,62 & 1 & 1,72 & 3 & 5,17 & 5 & 8,62 & 58 & 100,00 \\
\hline
\end{tabular}

Tabla 15. Nivel 19 Superior

\begin{tabular}{lrrrrrrrr}
\hline Materia prima & \multicolumn{2}{c}{ Cuarcita 1 } & \multicolumn{2}{c}{ Silex 1 } & \multicolumn{2}{c}{ Silex 2 } & \multicolumn{2}{c}{ Totales } \\
\hline Cuadricula & $N$ & $\%$ & $N$ & $\%$ & $N$ & $\%$ & $N$ & $\%$ \\
\hline N16 & 4 & 12,12 & - & - & - & - & 4 & 12,12 \\
N17 & 18 & 54,55 & 2 & 6,06 & 1 & 3,03 & 21 & 63,64 \\
N18 & 4 & 12,12 & 1 & 3,03 & 3 & 9,09 & 8 & 24,24 \\
\hline Muestra general & 26 & 78,79 & 3 & 9,09 & 4 & 12,12 & 33 & 100,00 \\
\hline
\end{tabular}

Microdesechos quemados

Respecto a este punto, es interesante destacar que se han registrado $(\mathrm{N}=19)$ solamente en sílex ( $\mathrm{S} 1)$ y los mismos se ubican en las cuadrículas N17 y N18 para el nivel $18 \mathrm{c}$ y en la N18 para el nivel 19 Superior (tabla 16).

Tabla 16. Distribución de Silex 1 quemado

\begin{tabular}{llccc}
\hline Nivel & Cuadrícula & Sector & N & $\%$ \\
\hline & N17 & 2 & 4 & 21,05 \\
& & 3 & 2 & 10,53 \\
\cline { 2 - 5 } $18 \mathrm{c}$ & 4 & 1 & 5,26 \\
\cline { 2 - 5 } & Total N17 & & 7 & 36,84 \\
\cline { 2 - 5 } & N18 & 1 & 3 & 15,79 \\
\cline { 2 - 5 } & Total N18 & 2 & 6 & 31,58 \\
\hline Total 18c & & & 9 & 47,37 \\
\hline 19 Superior & N18 & & 16 & 84,21 \\
\hline Muestra General & & & 19 & 15,79 \\
\hline
\end{tabular}


Distribuciones espaciales

Para comprender los procesos de formación del nivel Auriñaciense de El Castillo se implementaron (como se expuso anteriormente) diagramas de distribución de los microdesechos líticos por sector, cuadrícula y nivel (figuras 3 y 4). Como se puede observar en la distribución del nivel $18 \mathrm{c}$ (figura 3), la gran mayoria de los elementos se concentra en las cuadrículas N16, N17 y N18. En esta área destacan dos picos de densidad máxima que se ubican en la N16 y N18. Del resto de cuadrículas analizadas (J16, K12, K16, L16 y M16) también se observan dos picos de densidad, aunque de menor proporción que los anteriores, que se localizan en la $\mathrm{K} 16$ y en la L16. De igual modo es reseñable la abundante presencia de items en los sectores 7 y 8 de la cuadrícula M16 que vendrian a conformar conjuntamente con la N16 el mayor pico de densidad de ésta distribución. Por otra parte, en el nivel 19 Superior (figura 4), también se observa que la mayor presencia de microdesechos se ubican en las cuadrículas N16, N17 y N18 al igual que en el nivel 18 c (figura 3). En esta área se aprecia un pico de máxima densidad que se localiza en la cuadrícula $N 17$.

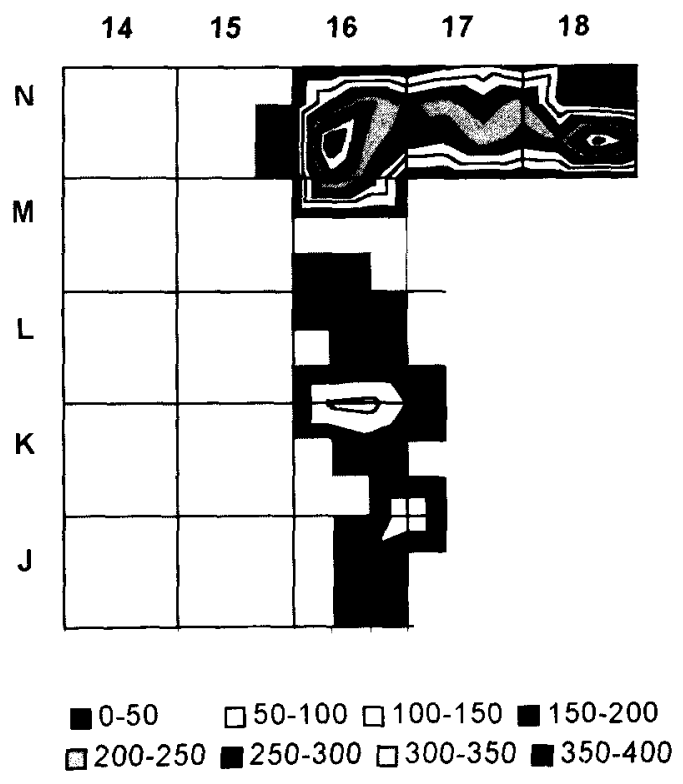

Figura 3. Distribución de microdesechos en el nivel $18 \mathrm{c}$ por cuadricula. 


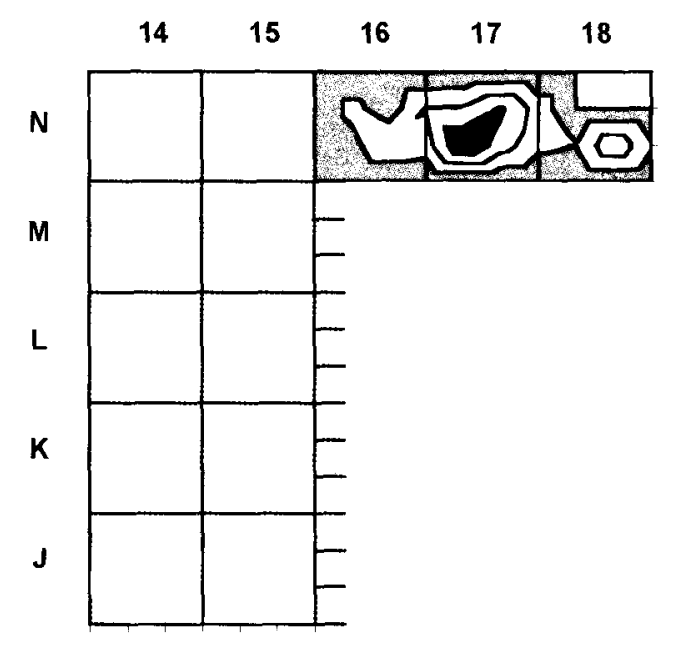

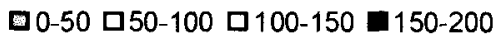

Figura 4. Distribución de microdesechos en el nivel 19 Superior por cuadrícula.

\section{DISCUSIÓN}

El análisis de los resultados permite realizar algunas inferencias acerca del conjunto de los microdesechos analizados. La identificación y análisis de las materias primas empleadas para la confección de conjuntos de útiles en los sitios arqueológicos proporcionan algunos aspectos relevantes que ayudan a discernir las estrategias tecnológicas desarrolladas por los grupos humanos. En este caso, sería la utilización mayoritaria de la cuarcita $(\mathrm{C} 1)$ frente a otras materias primas presentes en El Castillo. Esta observación coincide con en el análisis realizado por Cabrera Valdés et al., (1996) donde la C1 es la que predomina en el Auriñaciense Arcaico de este sitio. Sin embargo, se han observado diferencias:

En los útiles se registra limonita que no se constata en los microdesechos.

En los microdesechos se registra pizarra, arenisca, calcita y cuarzo; mientras que en los útiles de este nivel hay ausencia de estas materias primas.

Estas diferencias sugieren que las mencionadas materias primas (2) fueron trabajadas en el sitio y sus correspondientes útiles transportados a otras áreas. Por el contrario, la limonita, fue tallada en otra área e ingresada al sitio posteriormente. Esta situación ha sido mencionada anteriormente bajo los términos "Ghost" y "Orphans". 
La presencia de corteza junto a las lascas primarias y secundarias en el resto del conjunto permite inferir que en nivel $18 \mathrm{c}$ las materias primas pudieron haber entrado ya descortezada o en un estado avanzado de reducción en la que las lascas grandes y núcleos pudieron conservar parte de la corteza en alguna de sus caras (Cowan, 1999:604; Nash, 1996:86). Por su parte, la cuarcita y principalmente la $\mathrm{C} 1$ pudo haber ingresado en un estado de menor desbastado que el resto de las materias primas.

En lo que respecta al estado de fragmentación, el predominio en los distintos niveles de lascas indiferenciadas sumadas a las lascas fracturadas (con y sin talón) coinciden con lo propuesto por Ingbar et al., (1989:120-121) y Sullivan y Rozen (1985:762-763), en donde los desechos derivados de las actividades de talla de útiles alcanzan un porcentaje notoriamente mayor de indiferenciados junto a fracturados con y sin talón; del mismo modo, la proporción de núcleos y lascas enteras se reduce. Por lo tanto, es posible pensar que el conjunto de microdesechos de los niveles $18 \mathrm{c}, 18 \mathrm{c}$ Base y 19 Superior, está indicando que se han llevado a cabo actividades involucradas en la formatización, regularización y mantenimiento de utensilios.

El alto índice de fractura puede estar dado, también, por las actividades de talla con percutores duros ya que éstas generan más lascas fracturadas sin talón y con talones indiferenciados a diferencia del uso de percutores blandos y la talla por presión que producen más cantidad de lascas enteras (Patterson y Sollberger, 1978). De igual modo, este alto índice de fracturación podria provenir de la acción del pisoteo humano. Este factor ha sido constatado reiteradamente en varios estudios (ver entre otros a Clark et al.,1992; Clark, 1986; Gifford-Gonzalez et al., 1985; Maillo Fernández, 1998; Villa, 1982); los mismos proponen que cuando se producen las limpiezas de los talleres o lugares destinados a la talla de artefactos, se originan acumulaciones de materiales líticos donde el consiguiente pisoteo incrementa la fractura en los elementos.

Otros factores que intervienen en la fractura -aunque no detectable en El Castillo-serían la acción de los animales cavadores y la intensidad del pisoteo de otros animales (Bocek 1986; Erlandson, 1984; Gómez, 1996; Gómez et al., 1999; Hull, 1987; Gifford, 1978; Peretti y Curtoni, 1999; Villa, 1982). Finalmente, cabría añadir que la cuarcita durante el proceso de talla se fractura fácilmente, al tiempo que desprende un número elevado de astillas, esquirlas, y microdesechos (Nami, 1992 b). Por lo tanto, se infiere que el alto indice de fracturas detectado supra sobre la cuarcita, tiene relación con las condiciones particulares de la misma.

La tendencia observada en el tipo de lasca indica que se han desarrollado actividades relacionadas a la manufactura, mantenimiento $y / 0$ 
regularización de utensilios si se tiene en consideración la presencia de lascas angulares, planas y de reactivación directa. Estas últimos son claros indicadores de estas actividades y de la prolongación de la vida útil de los utensilios (Bamforth, 1986; Bellelli et al., 1985-1987; Binford, 1979; Shott, 1989). Las lascas de reactivación serán tratadas más adelante.

La información derivada del módulo de longitud/anchura refuerza la idea del desarrollo de actividades enfocadas a la manufactura, mantenimiento y/o regularización de útiles teniendo en cuenta la alta frecuencia de los mediano normal como subproductos de estas actividades. El análisis realizado por niveles no altera para nada estas inferencias. Por su parte, los resultados de los espesores permiten apoyar las ideas planteadas en lo concerniente a las actividades de talla mencionadas anteriormente.

De acuerdo a los resultados obtenidos en relación con los talones y bulbos se pueden extraer algunas conclusiones en el ámbito tecnológico. La elevada proporción de talones preparados en oposición a los no preparados evidencian claramente que las actividades orientadas a las últimas etapas de reducción se han desarrollado en mayor medida que las primeras etapas. Los talones preparados también son indicadores de estrategias conservadoras (Nash, 1996:88) ${ }^{3}$. Los bulbos, por su parte, presentan una alta frecuencia de difusos frente a los pronunciados. Ahora bien, mediante los resultados obtenidos de la combinación de los talones y bulbos se puede inferir algunas tendencias en las técnicas de talla como la aplicación de percutores blandos si se consideran los altos porcentajes de talones lisos y bulbos difusos, y la talla por presión caracterizada por los talones filiformes y puntiformes (Espinosa, 1995; Crabtree, 1972; Frison, 1968; Nami, 1991 a y b; Ohnuma y Bergman, 1982; Patterson y Silberger, 1978). Respecto a los talones corticales, las lascas fracturadas sin talón que presentan bulbos y los bulbos pronunciados se podria inferir, de igual modo, la aplicación de percutores duros (Baumler, 1985; Bergman y Roberts, 1988; Patterson y Sollberger, 1978) en menor medida que los blandos. Como hemos visto, en el análisis de estos dos atributos combinados por nivel y materias primas, no hay diferencias con respecto a las generalidades tecnológicas obtenidas en la representatividad de talones lisos y bulbos difusos; los bulbos pronunciados mantienen, también, una proporción constante independientemente del nivel y la materia prima. Por lo tanto, estos datos no alteran las conclusiones obtenidas.

No obstante, aunque estas inferencias a menudo sean razonables, no deberían tomarse como seguras puesto que no son unidades empíricas de observación. Desgraciadamente, la asociación de atributos con las técnicas específicas de reducción es rara vez exclusiva o determinista; estas 
asociaciones son tendencias estadísticas, no constantes (Nami y Bellelli, 1994; Shott, 1994; Teltser, 1991).

Los resultados derivados del estudio de las lascas de reactivación indican que en las cuadriculas (K16, L16, M16, N16, N17 Y N18) reflejan actividades de reactivación y mantenimiento de los filos en los utensilios (Binford,1979; Dibble, 1984; Fish, 1981; Kuhn, 1991; Stevenson, 1985; entre otros), preferentemente sobre cuarcita y silex en relación con el resto de las materias primas líticas. Teniendo en cuenta las conclusiones obtenidas acerca del atributo tipo de lasca junto con la información derivada de las lascas de reactivación, se infiere que no solo se han llevado a cabo este tipo de actividades sino que hay una clara preferencia a mantener los útiles en estas materias primas, frente a las demás.

Al respecto, es importante tener en cuenta que, dentro de un mismo grupo humano se plantean diversos sistemas de manufactura, con respecto a la materia prima, distancia a las fuentes de aprovisionamiento, funcionalidad de los utensilios a obtener y cuestiones sociales e ideológicas (Criado, 1993; Hernando, 1999; Hodder, 1988; Gamble, 1993; Gero, 1989; Gould, 1980; Ingold, 1993; MacBryde, 1984; Nash, 1996; Shennan, 1982; White y Modjesca, 1978; entre muchos más) '. Un ejemplo clásico para esto es el trabajo de Binford con los Esquimales Nunamiut en donde observó que la manufactura y el mantenimiento se realizan en las bases residenciales para la mayoría de las clases de utensilios que tienen una tarea específica (Binford, 1978). Por otra parte, se debería considerar también, que el mantenimiento y el reciclado de útiles se relaciona estrechamente con la disponibilidad de materia prima y no directamente con la organización del asentamiento o con los limites de tiempo de las actividades dentro de las cuales los artefactos eran usados (Bamforth, 1986; Torrence, 1989; Pokotylo y Hanks, 1989).

Por lo tanto, si se considera que las tecnologías conservadoras (curated) producen conjuntos que son tecnológicamente sofisticados y probablemente distintos en su forma, donde los utensilios individuales serán destinados para una variedad de propósitos anticipados, mantenidos para un número indeterminado de usos, transportados entre yacimentos para estos usos y reciclados para otras tareas (Bamforth, 1986:38; Binford, 1979:269,270; Shott, 1989:24) se podría pensar que en El Castillo se ha dado prioridad a las estrategias tecnológicas conservadoras en detrimento de las expeditivas (expedient), considerando que la industria lítica del nivel adelante.

El concepto de estrategias tecnológicas conservadoras y expeditivas será tratado más 
$18 \mathrm{c}$ se caracteriza, en primer lugar, por utensilios con un mayor grado de inversión de energía en su elaboración (Bamforth, 1986:38) y esta compuesto por una elevada representatividad del grupo de raspadores (66 en cuarcita, 10 en sílex, 2 en ofita y 1 en limonita), seguidos por el grupo de piezas retocadas (raederas y piezas con retoque contínuo; 86 en cuarcita, 12 en sílex y 3 en ofita), puntas ( 4 en cuarcita y 1 en sílex), limaces ( 1 en sílex), perforadores ( 1 en cuarcita), buriles ( 14 en cuarcita y 1 en sílex) y, por último, el grupo de útiles compuestos (piezas retocadas que a su vez presentan una muesca, un raspador $u$ otro útil; 4 en cuarcita y 1 en ofita) (Cabrera Valdés et al., 1996).

Teniendo en cuenta, que el grupo mayoritario es el de los raspadores y, que estos, tienen la particularidad de poder ser usados para diversas tareas, refuerza aún más la idea de estrategias tecnológicas conservadoras planteadas más arriba. En segundo lugar, se encuentran un conjunto de útiles que tienen un menor costo energético en su elaboración lo que implicaría estrategias tecnológicas expeditivas que producen conjuntos que son tecnológicamente más simples y la manufactura de utensilios sería una respuesta inmediata a tareas específicas y descartados posteriormente a su uso (Binford, 1979:269; Bamforth, 1986:38). Estos útiles se encuentran comprendidos por el grupo de piezas escotadas (4 en cuarcita y 1 en ofita), seguidas por el grupo de piezas denticuladas ( 3 en cuarcita), el grupo de armaduras (1 en cuarcita), el grupo de piezas truncadas (1 en cuarcita). De la totalidad de utensilios ( $\mathrm{N}=216)$, la materia prima más representada es la cuarcita $(84,72 \%)$, seguida por el sílex $(11,57 \%)$, la ofita $(3,24 \%)$, y la limonita $(0,46 \%)$. Como se puede observar, las materias primas en que han sido manufacturados los diferentes utensilios son preferentemente cuarcita y sílex locales y, en menor medida, ofita y limonita (Cabrera Valdés et al., 1996; Cabrera Valdés y Bernaldo de Quirós, 1996). Sin embargo, a pesar de que las estrategias conservadoras y expeditivas se han constatado en esta yacimiento, éstas no deberían considerarse como únicas y excluyentes según lo propuesto por Nelson (1991), en donde el oportunismo (opportunistic) no esta planificado y responde a condiciones inmediatas y no anticipadas (Nelson, 1991:62-66).

Por otra parte, los hallazgos de microdesechos quemados en sílex sugieren que podrían ser el resultado de las actividades de limpieza llevadas a cabo en los hogares y posteriormente transportados y depositados en éstas áreas. Esta observación, apoya lo expuesto acerca de este tema por Cabrera Valdés y Bernaldo de Quirós (1996:135).

De las distribuciones espaciales diferenciales descritas, se desprenden las siguientes observaciones: 
1. Los picos y zonas de elevada densidad observadas en la figura 3 podrian estar representando, por un lado, acumulaciones de carácter probablemente primario derivadas de las actividades de talla in situ (Gould 1981; Moholoy-Nagy 1990) y acumulaciones secundarias producto de las actividades de limpieza, por el otro.

2. Estas concentraciones de microdesechos líticos también pueden deberse en parte a los procesos naturales que actuaron en el nivel 18c.

3. La distribución observada puede estar conformada, de este modo, sobre la base del tamaño del material pudiendo estar o no relacionada con la función o las actividades realizadas, expuestas a procesos postdeposicionales.

4. La distribución del nivel 19 Superior responde a patrones similares al nivel anterior. Si se considera la esterilidad de este nivel (Cabrera Valdés et al.,1993:87), la concentración identificada (especialmente en la cuadrícula N17) responde fundamentalmente al pisoteo del nivel en la época Auriñaciense y a la presión sedimentaria provocada por la gran potencia estratigráfica de los niveles más recientes. En relación a esto, se puede inferir también, que los microdesechos de este nivel pudieron haber conformado junto con los del nivel $18 \mathrm{c}$ una distribución uniforme de los mismos en las cuadrículas N16, N17 y N18.

Para ampliar estas observaciones, se debe destacar que la cantidad y tipo de los microdesechos puede ser comparado con la cantidad y tipo de los instrumentos líticos del mismo contexto, recogidos a través de las excavaciones, y entonces pueden ser identificados varios patrones de conducta. El reconocimiento de tales patrones es posible a través del uso de información sobre hábitos de desechos contemporáneos, mediante la aplicación de estudios actualísticos apropiados para áreas específicas (Hull, 1987:773).

En tal sentido, en un estudio de hábitos de disposición contemporánea entre los Nunamiut, Binford (1978) ha observado que los objetos grandes tienden a ser sacados fuera del área inmediata de uso, mientras que los objetos pequeños fueron dejados en el sitio. También observó que las áreas de actividad tienden a ser reusadas para tareas específicas, provocando una acumulación de objetos pequeños en áreas usadas con exceso, mientras que la acumulación secundaria compuesta de objetos grandes se sitúa en áreas de uso poco frecuente.

Es válido tener en cuenta que las ocupaciones intensivas, como en El Castillo, pudieron contribuir a la preservación de los microdesechos líticos a través del pisoteo creciente (McBrearty et al., 1998; Villa, 1982), evitando la 
alteración postdepositacional producida por los agentes naturales (i.e. transporte eólico, acción del agua, entre otros) que pudieran crear nuevos patrones depositacionales (Fladmark, 1982). Por otro lado, el pisoteo puede producir la migración de los objetos más pequeños, a diferencia de los grandes, hacia distintas profundidades que pueden variar dependiendo de la matriz sedimentaria (Stockton, 1973; Yellen, 1977; Gifford y Behrensmeyer, 1977), como en el caso del nivel 19 Superior. Esta situación de alteración postdeposicional podría existir tanto en depósitos al aire libre como en cuevas y abrigos, con independencia de la textura de las matrices sedimentarias (Stockton, 1973, 1977) Según Villa (1982) esta alteración podría ser contemporánea al entierro de los elementos.

Los estudios etnoarqueológicos realizados por D. Clark; N. Toth y $\mathrm{G}$. Ligabue (1992), con los grupos horticultores Langda de las tierras altas de Nueva Guinea, proporcionan datos importantes acerca de las actividades de talla que realizan los hombres de la aldea. Estos, junto a sus hijos, fabrican hachas que luego serán usadas para talar árboles y cortar maderas. La manufactura de las mismas consta de tres etapas bien definidas. A cada una le corresponde una morfologia de hacha y unos restos de talla característicos. Estos últimos tienden a concentrarse en los lugares en que fueron realizadas las tres etapas. Una particularidad de estas actividades, es que los talladores trabajan solos o en grupo. Si se trabaja en grupo, se sientan siempre en línea y mirando en la misma dirección, de modo que los pequeños desechos que salten no lastimen a ninguno. Estos residuos suelen encontrarse fuera del perímetro del área de trabajo o alrededor de una habitación y terminan esparcidos de forma circular u oval en un área de un metro cuadrado. La dispersión posterior de los mismos se debe al tránsito de personas $o$ animales que producen el entierro cuando hay barro. Para los autores, los desechos de cada etapa de manufactura reflejarían pautas conservadas en el registro arqueológico.

En este sentido, es interesante destacar también, los estudios experimentales de Behm (1983) con respecto a las concentraciones de microdesechos, en ellos demuestra que en los depósitos primarios se concentran según patrones circulares u ovales, de igual manera demuestra que los elementos tienden a concentrarse directamente enfrente del tallador. La práctica de limpieza de estas concentraciones formará depósitos secundarios en los que se incluirian también los desechos más grandes. Estos depósitos son fáciles de distinguir observando las diferencias de tamaño y forma de los elementos.

Con respecto a los procesos postdeposicionales, algunos estudios indican que los materiales pequeños están depositados y redepositados en 
forma diferente respecto de los materiales grandes (Villa, 1982:278-279). Esto puede deberse a factores tales como: 1) la actividad de los roedores cuyo efecto se considera perturbador y destructivo (Bocek, 1986 y 1992; Erlandson, 1984; Gómez, 1996, Gómez et al., 1999), sin embargo, creemos que este agente no ha influido en el yacimiento, porque en el curso de las excavaciones no se han detectado galerías correspondientes a roedores (Cabrera Valdés, com. pers.); 2) el crecimiento de las raíces que puede llegar a mezclar los materiales (Schiffer, 1987); 3) el efecto del clima, que produce dinámicas como distintas velocidades del viento, lluvia, congelamiento y descongelamiento en la superficie de los depósitos arqueológicos pudiendo afectar en parte los mismos (Laville, 1969; Schiffer, 1987); 4) el agua puede ser causante del desplazamiento horizontal de los materiales y este factor se puede reconocer por el estado físico (rodado), incluso se puede apreciar si existen concentraciones de materiales en zonas donde es previsible que se hallan producido escorrentías o canales (Glover, 1979; Peretti y Curtoni, 1999). No obstante, esta situación no se ha corroborado, en este análisis, con la presencia de materiales rodados y con los estudios estratigráficos exhaustivos realizados por Cabrera Valdés et al., (1993); 5) la alternativa humedad y secado de las matrices sedimentarias pueden producir migraciones verticales (Cahen y Moeyersons, 1977); 6) la reptación, solifluxión y la subsidencia de depósitos en cueva produce desplazamientos postdeposicionales (Rick, 1976; Freeman, 1975; Jelinek et al., 1973; Glover, 1979).

\section{CONSIDERACIONES FINALES}

A modo de síntesis, puede decirse que El Castillo presenta un registro temporalmente extenso de las ocupaciones humanas en el área Cantábrica. En especial, la evidencia procedente de este sitio permite discutir diversos aspectos vinculados a la transición del Paleolítico Medio al Superior, tales como las estrategias tecnológicas, la economía de los habitantes humanos del área, y la naturaleza de las relaciones sociales dentro y entre los grupos cazadores-recolectores. Del mismo modo, permite discutir la gran variabilidad existente en las industrias líticas y óseas, así como realizar inferencias acerca de los factores ecológicos, ideológicos y simbólicos que se hallan en su origen (Bernaldo de Quirós y Cabrera Valdés, 1996).

Como se ha propuesto al comienzo de este trabajo, se han analizado los microdesechos líticos provenientes del nivel Auriñaciense Arcaico de la Cueva de El Castillo. El análisis y las conclusiones que se derivan del mismo deben considerarse preliminares, hasta que se puedan integrar los 
resultados aquí obtenidos con la totalidad de los microdesechos líticos recuperados en los demás niveles involucrados en la transición del Paleolítico Medio al Superior. Esta es la razón que impide formular conclusiones de órden más general relativas a las actividades realizadas en el sitio o a la dedicación funcional en cada una de las áreas. No obstante, a pesar de ello, la información que se ha obtenido con el porcentaje de los materiales líticos estudiados respecto del conjunto total del sitio es muy relevante.

En consecuencia, este estudio se ha basado, por un lado, en tratar de aislar factores culturales y naturales que pudieron haber alterado el depósito arqueológico del yacimiento $y$, por el otro, en los procesos técnicos que no son más que la producción de objetos diferentes, siendo las actividades de talla lítica una parte esencial del proceso productivo de los grupos humanos. Por lo tanto, el análisis de los microdesechos líticos es una contribución que permite entender de una manera más precisa la dinámica de formación del registro arqueológico y orienta a comprender y explicar las actividades y procesos que derivan del subsistema de producción lítica.

De acuerdo con lo expuesto en este trabajo, el análisis efectuado con las materias primas líticas permitió establecer que el orden de la frecuencia de éstas es el mismo que se registra para todo el sitio, ya que en los estudios anteriores se observaron frecuencias más altas de cuarcita (C1) respecto de las otras materias primas (i.e. silex, ofita, limonita, entre otras). También, fue observado un bajo índice de corteza, lo que indicaría que las primeras etapas de la secuencia de producción lítica pudieron haber sido realizadas en otra área o que los materiales más grandes entraran en un estado avanzado de reducción conservando parte de la corteza en alguna de sus caras; y que, al menos la cuarcita (C1) pudo haber ingresado al yacimiento en un estado de menor desbastado que el resto de las materias primas.

En cuanto al análisis realizado con los atributos tecnológicos (i.e. talones, bulbos, tipo de lasca, módulo longitud anchura, etc.), se han podido establecer a través de los mismos que en el nivel $18 \mathrm{c}$ se han llevado a cabo actividades de manufactura, uso, modificación y mantenimiento opcional de los utensilios y tendencias en las técnicas de talla referentes a la aplicación de percutores blandos en mayor medida que los duros, así como, la aplicación de la técnica por presión. Los resultados obtenidos del estado de fragmentación sugieren el desarrollo de las mismas actividades mencionadas anteriormente. En este sentido, el alto índice de fracturación también puede provenir de la acción del pisoteo humano y la intensidad del pisoteo de otros animales en las áreas de actividad de talla. Éstas, después de ser barridas o limpiadas, tienden a formar sub-concen- 
traciones de microdesechos líticos entre los cuales aumentaría el índice de fracturación. Por otro lado, se debe reseñar que aunque la cuarcita presenta cualidades excelentes para la talla tiende a fracturarse fácilmente y en general, durante el proceso de talla se desprenden una cantidad considerable de lascas pequeñas, microdesechos, astillas y esquirlas. Por su parte, la localización en un área específica del sitio de microdesechos quemados en sílex (S1) nos ha permitido sugerir que los mismos provengan de las actividades de limpieza de otras áreas. Otro de los puntos importantes sería el mantenimiento preferencial de los utensilios elaborados en cuarcita $(\mathrm{C} 1)$ y sílex frente a las demás materias primas. También, se han constatado diferencias en la manufactura de los útiles, observándose una preferencia de estrategias tecnológicas conservadoras en detrimento de las expeditivas.

En cuanto a los mapas de densidad, se pudo observar una distribución espacial diferencial de los microdesechos líticos. Estos estarian reflejando en un área determinada del sitio acumulaciones de carácter probablemente primario formadas por la concentración de desechos correspondientes a la actividad de talla in situ, por un lado, y acumulaciones de carácter secundario, producidas por las actividades de limpieza, por el otro.

También se consideró la posibilidad de que estas acumulaciones fueran el efecto de los distintos procesos postdeposicionales. En este sentido, es interesante destacar que los elementos contenidos en el nivel 19 Superior se han depositado en el mismo debido a la presión sedimentaria por la gran potencia estratigráfica de los niveles más recientes y al pisoteo. los microdesechos de éste nivel, junto con los del $18 \mathrm{c}$, podrian haber conformado una distribución uniforme en las áreas más densamente representadas.

Teniendo en cuenta, los resultados preliminares obtenidos, se plantea la necesidad de evaluar de una manera más profunda qué agentes fueron la causa de la configuración de las distribuciones espaciales observadas en los distintos niveles de El Castillo. Por lo tanto, aparte de estudiar todos los materiales que provienen de otros niveles culturales, siguiendo la metodología implementada en este trabajo, es necesario aplicar diseños de experimentación para comprender y estimar el grado de incidencia que tienen los distintos agentes culturales y naturales responsables de la formación del depósito arqueológico de este yacimiento.

Considerando lo expuesto en este trabajo, se puede afirmar que el análisis de microdesechos líticos es importante, porque ha permitido abordar e interpretar, de una manera más profunda, los procesos de formación del depósito arqueológico de la Cueva de EI Castillo. 


\section{AGRADECIMIENTOS}

Los autores desean agradecer especialmente a los Dres. Victoria Cabrera Valdés y Federico Bernaldo de Quirós por su predisposición, contribución y sugerencias a lo largo de todo el trabajo.

También queremos agradecer:

Al Dr. Gustavo Martínez; a los Lic. Gustavo Gómez, José Manuel Maíllo Fernández y Paula Barros por los comentarios sobre la primera versión de este manuscrito.

A los Dres. María Teresa Alberdi, Sergio Sánchez y Enrique Sanz por colaboración en el Museo de Ciencias Naturales de Madrid.

A Mila Montes Pérez, secretaria del Dpto de Prehistoria e Historia Antigua de la UNED por haber estado siempre dispuesta a solucionarnos cuestiones de distinta índole.

A Torsten Frenk, Patrick Wiik y Paco Calderón por su ayuda desinteresada.

A todo el equipo de El Castillo que de manera anónima ha cribado y triado los sedimentos durante muchos años.

Este trabajo ha sido posible gracias al Programa VII, Facultad de Ciencias Sociales, UNC, Olavarría (Argentina), asi como de la Beca Predoctoral de la UNED Madrid (España).

Los errores que se puedan encontrar en el trabajo son de nuestra exclusiva responsabilidad.

\section{BIBLIOGRAFIA}

ASCHER, R. (1961): Experimental Archaeology. American Anthropologist 63:793-816.

AsCHERo, C. (1975): Ensayo para una Clasificación Morfológica de Artefactos Líticos aplicada a Estudios Tipológicos Comparativos. Informe presentado al CONICET. Buenos Aires. MS.

- (1983): Ensayo para una Clasificación Morfológica de Artefactos. Apéndice $A$ y $B$. Cátedra de Ergología y Tecnología. Facultad de Filosofía y Letras. Universidad de Buenos Aires. MS.

BagoliNI, B. (1968): Ricerche sulle Dimensioni dei Manufatti Litici Prehistorici non Ritocatti. Annali dell' Universita di Ferrara. Nuova Serie. Sezione XV, vol. I, N. ${ }^{\circ} 10: 195-219$. Ferrara.

BAMFORTH, D. (1986): Technologycal Efficiency and Tool Curation. American Antiquity 51 (1): 38-50.Baumler, M. (1985): On the interpretation of Chipping Debris Concentrations in the Archaeological Record. Lithic Technology, 14 (3):120-125.

BaumLeR, M. y C. Downum. (1989): Between Micro and Macro: a Study in the Interpretation of Small-Size Lithic Debitage. Experiments in Lithic Technology. Amick, E. (Ed). B. A. R. International Series, 528:101-116

BEHM, J. (1983): Flake Concentrations: Distinguishing between Flintworking Activity Areas and secondary deposits. Lithic Technology 12(1): 9-16. 
Bellelli, C., G. Guraieb y J. Garcia. (1985-1987): Propuesta para el Análisis y Procesamiento por Computadora de Desechos de Talla Lítica (DELCO. Desechos Líticos Computarizados). Arqueología Contemporánea 2(1):36-53. Buenos Aires.

Bergman, C. y M. Roberts (1988): Flaking Techniques at the Acheulean Site of Boxgrove, West Sussex (England). En Cultures et Industries Litiques en Milieu Loessique. A. Tuffreau (Ed). Revue Archéologique de Picardie n. ${ }^{\circ}$ 1-2: 105-112. Amiens, France.

Bernaldo de Quirós, F. y V. Cabrera Valdés (1996): Economical Strategies in the Upper Paleolithic in the Cantabrian region. Human Evolution 11(2): 121-128.

BiNFORD, L. (1978): Dimensional Analysis of Behavior and Site Structure: Learning from Eskimo Hunting Stand. American Antiquity 43:330-361.

- (1979): Organization and Formation Processes: Looking at Curated Technologies, Journal of Anthropological Research 35 (3):255-273.

BOCEK, B. (1986): Rodent Ecology and Burrowing Behavior: Predicted Effects on Archaeological Site Formation. American Antiquity 51(3):589-603.

BISCHOFF, J, N. Soler, J. MAROto y R. JULIA. (1989): Abrupt Mousterian/Aurignacian Boundary at ca. $40 \mathrm{Ka} \mathrm{bp:} \mathrm{Accelerator} \mathrm{Radiocarbon} \mathrm{Dates} \mathrm{from} \mathrm{L'Arbreda} \mathrm{Cave} \mathrm{(Catalunya,} \mathrm{Spain).}$ Journal of Archaeological Science 16:563-576.

Bischoff, J, K. Ludwig, J. F. Garcia, E. Carbonell, M. Vaquero, T. Stafford y A. Julia (1994): Dating of the Basal Aurignacian Sandwich at Abric Romani (Catalunya, Spain) by Radiocarbon and Uranium-Series. Journal of Archaeological Science 21:541-551.

BocEk, B. (1992): The Jasper Ridge Reexcavation Experiment: Rates of Artifact Mixing by Rodents. American Antiquity 57(2):261-269.

Cabrera Valdés, V. y F. Bernaldo de Quirós (1996): El Hombre Moderno en Cantabria: La Transición del Paleolítico Medio al Paleolítico Superior a través de la Revisión Estratigráfica de la Cueva del Castillo. Actas del Primer Encuentro de Historia de Cantabria. Universidad de Cantabria y Gobierno de Cantabria. Santander. Tomo1: 129-147.

CABRERA VALDÉs, V. y J. BISCHOFF. (1989): Accelerator Radiocarbon Dates for EarlyUpper Paleolithic (Basal Aurignacian) at El Castillo Cave (Spain). Journal of Archaeological Science 16:577-584.

Cabrera Valdés, V., M. Hoyos y F. Bernaldo de Quirós (1993): La Transición del Paleolítico Medio / Paleolítico Superior en la Cueva de El Castillo: Características Paleoclimáticas y Situación Cronológica. El Origen del Hombre Moderno en el Suroeste de Europa. V. Cabrera (Ed). Universidad Nacional de Educación a Distancia. Madrid. 81-101.

Cabrera Valdés, V, M. Lloret Martinez de la Riva y F. Bernaldo de Quirós (1996): Materias Primas y Formas Líticas del Auriñaciense Arcaico de la Cueva del Castillo (Puente Viesgo, Cantabria). Espacio, Tiempo y Forma. Serie 1, Prehistoria y Arqueologia 9: 141-158.

CAHEN, D y J. MOEYERSONS (1977): Subsurface Movements of Stone Artifact and Their Implications for the Prehistory of Central Africa. Nature 266: 812-815.

CLARK, J. (1986): Another Look at Small Debitage and Microdebitage. Lithic Technology 15:21-33.

Clark, D., G. Ligabue y N. TOth (1982): Los Últimos Fabricantes de Hachas de Piedra. Investigación y Ciencia 192:6-11.

Collins, M. (1975): Lithic Technology as a Means of Processual Inference. Lithic Technology: Making and Using Stone Tools. E. Swanson (Ed.). 5-34. Mouton Publishers. The Hague.

- (1989-1990): Una Propuesta Conductual Para el Estudio de la Arqueología Lítica. Etnia 34-35:47-65. Olavarría.

Cowan, F, (1999): Making Sense of Flake Scatters: Lithic Technological Strategies and Mobility. American Antiquity 64 (4) :593-607.

Crabtree, D. (1972): An Introduction to Flintworking. Occasional Papers of the Idaho State University Museum, 28.

Criado, F. (1993): Visibilidad e Interpretación del Registro Arqueológico. Trabajos de Prehistoria $50: 39-56$.

DEAL, M. (1985): Household Pottery Disposal in the Maya High-lands: An Ethnoarchaeological Interpretation. Journal of Anthropological Archaeology :243-291.

Dibble, H, (1984): Interpreting Typological Variations of Middle Paleolithic Scrapers: Functions, Style or Sequence of Reduction?. Journal of Field Archaeology 11:431-436.

DUNNELL, R., y J. STEIN. (1989): Theoretical Issues in the Interpretation of Micro-artifacts. Geoaechaeology 4:31-42. 
Ericson, J. (1984): Toward the Analysis of Lithic Production Systems. Prehistoric Quarries and Lithic Productions. J. Ericson y B. Purdy (Eds), 1-9. Cambridge University Press. Cambridge.

ERLANDSON, J. (1984): A Case Study in Faunalturbation: Delineating the Effects of the Burrowing Pocket Gopher on the Distribution of Archaeological Materials. American Antiquity 49 (4): 785-790.

EsPinOSA, S. (1995): Dr. School y Monsier Fleur: de Talones y Bulbos. Cuadernos 16 :315328. Instituto Nacional de Antropología y Pensamiento Latinoamericano. Secretaría de Cultura de la Nación. Buenos Aires.

FISH, P. (1981): Beyond Tools: Middle Palaeolithic Mebitage Analysis and Cultural Inference. Journal of Anthropological Research 37:374-386.

FLADMARK, K.(1982): Microdebitage Analysis: Initial Considerations. Journal of Archaeological Science 9:205-220.

Freeman, L. (1975): Acheulian Sites and Stratigraphy in Iberia and the Magreb. In After the Australopithesines. K. Butzer and G. Isaac (Eds). 661-743. Mouton. The Hague.

Frison, G, (1968): A Functional Analysis of Certain Chipped Stone Tools. American Antiquity 33: $149-155$.

Gallagher, J. (1977). Contemporary Stone Tools in Ethiopia: Implications for Archaeology. Journal of Field Archaeology 4: 407-414.

Gamble, C. (1993): Exchange, Foraging and Local Hominid Networks. Trade and Exchange in Prehistoric Europe, C. Scarre y F. Healy (Eds). 35-44. Oxbow Monograph 33. Oxbow Books, Oxford.

GERO, J. (1989): Assessing Social Information in Material Objects: How Well Do Lithics Measure Up?. Time Energy and Stone Tools, R. Torrence (Ed). 92-105. Cambridge University Press.

GifFORD, D. (1978): Ethnoarchaeological Observations on Natural Processes Affecting Cultural Materials. Explorations in Etnoarchaeology. R. Gould (Ed.). 77-102. University of New Mexico Press. Albuquerque.

GifFORD, D y K. BeHRENSMEYER (1977): Observed Formation and Burial of Recent Human Occupation Site in Kenya. Cuaternary Research 8: 245-266.

Gifford-Gonzalez, D., D. B. Damrosh, D.R. Damrosh, J. Pryor y R. Thunen (1985): The Third Dimension in Site Structure: an Experiment Trampling and Vertical Dispersal. American Antiquity 50 (4): 803-818.

GLover, I (1979): The Effects of Sink Action of Archaeological Deposits in Caves: and Indonesian Example. World Archaeology 10: 302-317.

Gómez, G. (1996): Los Pequeños Mamíferos del Sitio Arroyo Seco 2 (Ptdo. de Tres Arroyos, Pcia. de Bs. As. Aspectos Relacionados con la Subsistencia, Tafonomía y el Paleoclima. Tesis de Licenciatura. Facultad de Ciencias Sociales. Universidad Nacional del Centro. Olavarría. MS.

Gómez, G, J.L. Prado y M. T. Alberdi (1999): Micromamíferos del Sitio Arroyo Seco 2 (Provincia de Buenos Aires, Argentina). Sus Implicaciones Tafonómicas y Paleoambientales. Estudios Geológicos 55(5-6): 273-281.

Gould, R. (1979): Exotic Stones and Battered Bones. Ethnoarchaeology in the Australian Desert. Archaeology 32:229-237.

- (1980): Living Archaeology. Cambridge University Press. USA.

- (1981): Brandon Revisted: A New Look at an Old Technology. En Modern Material Culture: The Archaeology of Us, R. Gould y M. B. Schiffer (Eds.). 269-281. Academic Press. New York.

HASSAN, F.(1978): Sediments in Archaeology: Methods an Implications for Paleoenvironmental and Cultural Analysis. Journal of Field Archaeology 5:197-213.

Hernando, A. (1999): El Espacio no es Necesariamente un Lugar: En Torno al Concepto de Espacio y a sus Implicaciones en el Estudio de la Prehistoria. Arqueología Espacial 21:7-27. Teruel, España.

Hodder, I. (1988): Interpretación en Arqueologia. Corrientes Actuales. Crítica, Barcelona.

Hoyos, M. (1979): El Karst en Asturias en el Pleistoceno Superior y Holoceno. Tesis Doctoral. Universidad Complutense. Madrid.

HULL, K. (1987): Identification of Cultural Site Formation Processes Through Microdebitage Analysis. American Antiquity 54: 851-855.

INGBAR, E., M. LARSON y B. BRADLEY. (1989): A None Tipologycal Approach to Debitage Analysis. In Amick, D y MAULoING (Eds). Experiments in Lithic Technology. British Archaeologycal Reports International Series 528: 17-135. Oxford. 
INGLOD, T. (1993): The Temporality of the Landscape. World Archaeology 25 (2):152-174.

Jelinek, A., W. Farrand, G. HaAs, A. Horowitz y P. Goldoberg (1973): New excavations at the Tabun Cave. Mount Carmel. Israel. 1967-1972: a Preliminary report. Paléorient 1: 151-183.

KUHN, S. (1991): "Unpacking" Reduction: Lithic Raw Material Economy in the Mousterian of West-Central Italy. Journal of Anthropological Archaeology 10:76-106.

LANGe, F y C. Rydberg. (1972): Abandonment and Post-abandonment Behavior at a Rural Central American House Site. American Antiquity. 37:419-432.

LAVILLE, H. (1969): L'Interestade Wurm II- Wurm III et la Position Chronologique du Paléolithique Supérieur Ancien en Périgord. Comptes Rendus de L'Academie des Sciences. París 269: 10-12.

MAdSEN, M. (1992): Lithic Manufacture at British Camp: Evidence From Size Distributions and Microartifacts. Stain ,J. (Ed.). Deciphering a Shell Midden. Academic Press, San Diego, 193-210.

MACBRYDE, I. (1984): Kulin Greenstone Quarries: the Social Contexts of Production and Distribution for the Mt. William Site. World Archaeology 16 (2): 267-285.

McBrearty, S, L. Bishop, T. Plummer, R. Dewar y N. Conard (1998): Tools Underfoot: Human Trampling as an Agent of Lithic Artifact Edge Modifications. American Antiquity 63(1):108129.

MCKeLLAR, L. (1983): Correlation and Explanation of Distributions. Atlatl: Occasional Papers: $n{ }^{\circ}$ 4. Department of Anthropology, University of Arizona, Tucson.

Maillo Fernández, J. (1998): Proporciones de Debris en Réplicas de Talla Experimental. Espacio, Tiempo y Forma, Serie 1, Prehistoria y Arqueologia, 11:45-55.

Moholoy-NAGY, M. (1990): The Misidentification of Mesoamerican Lithic Workshop. Latin American Antiquity 1:268-279

MoRRow, T. (1996): Lithic Refitting and Archaeological Site Formation Processes. A case Study from the Twin Ditch Site, Greene County, Illinois. ?. Stone Tools Theoretical Insights into Human Prehistory. G. Odell (Ed). 345-373. Plenum Press. New York \& London.

Nami, H. (1991)a: Desechos de Talla y Teoría de Alcance Medio: un Caso de Península Mitre, Tierra del Fuego. Shincal 3(2):94-112. Actas X Congreso Nacional de Arqueología Argentina. San Fernando del Valle de Catamarca.

- (1991)b: Algunas Reflexiones Teóricas sobre Arqueología y Experimentación. Shincal 3(1):151-168. Actas $X$ Congreso Nacional de Arqueología Argentina. San Fernando del Valle de Catamarca.

Nami, H. (1992)a: Informe sobre el Primer Curso de Análisis de Desechos de Talla Experimentales en Argentina. Palimpsesto. Revista de Arqueología 1:75-79.

- (1992)b: El Subsistema Tecnológico de la Confección de Instrumentos Líticos y la Explotación de los Recursos del Ambiente: una Nueva Vía de Aproximación. hincal. 2:33-53. Universidad Nacional de Catamarca, Argentina.

Nami, H. y C. Bellelli. (1994): Hojas, Experimentos y Análisis de Desechos de Talla. Implicaciones Arqueológicas para la Patagonia Centro-Septentrional. Cuadernos del Instituto Nacional de Antropología 15:199-223. Buenos Aires.

Nami, $\mathrm{H}$ y J. Rabassa. (1988): Experimentos, Petrografía y Confección de Instrumentos de Piedra con Ignimbritas. Pilcaniyeu. Observaciones para el Conocimiento de las Sociedades del Pasado. Revista Estudios Regionales 2:131-148. Mendoza.

NASH, S. (1996): Is Curation Useful Heuristic?. Stone Tools Theoretical Insights into Human Prehistory. G. Odell (Ed). 81-99. Plenum Press. New York \& London.

NeLson, M. (1991): The Study of Technological Organization. Archaeological Method and Theory. M. B. Schiffer (Ed). Vol 3:57-100. Tucson, Arizona.

NiCHOLSON, B. (1993): A Comparative Evaluation of Four Sampling Techniques and of the Reliability of Microdebitage as a Cultural Indicator in Regional Surveys. Plains Anthropologist 28: $273-281$.

Nielsen, A. (1994): Como es Arriba es Abajo: Evaluación Crítica de las Posibilidades del Análisis de Microartefactos para la Inferencia Arqueológica. Arqueología 4:9-37. Instituto de Ciencias Antropológicas. Universidad de Buenos Aires. Buenos Aires.

ODELL, G. (1980): Toward a more Behavioral Approach to Archaelogical Lithic Concentrations. American Antiquity 45 (3): 404-431.

Ohnuma, K. y C. Bergman (1982): Experimental Studies in the Determination of Flaking Mode. Boulletin of the Institute of Archaeology 19: 161-170. University of London. 
Patterson, L. y B. Sollberger (1978): Replication and Classification of Small Size Lithic Debitage. Plains Anthropologist 23(80):103-112.

PERETTI, R. (1997): Estudio de Microdesechos Líticos en el Sitio Arqueológico Arroyo Seco 2 (Pdo. De Tres Arroyos. Pcia. De Buenos Aires): Nuevas Vias de Aproximación a la Comprensión del Subsistema Tecnológico Lítico. Tesis de Licenciatura. Facultad de Ciencias Sociales. Universidad Nacional del Centro. Olavarria. MS.

PERETTI, R. y R. CURTONi (1999): Entraron Enteras y salieron Rotas: Lascas Experimentales y Procesos de Formación en Conjuntos Líticos. MS.

PoKoTYLO, D. y C. HANKS. (1989): Variability in Curated Lithic Technology: An Ethnoarchaeologycal Case Study from the Mackenzie Basin, Northwest Territories, Canada.), Experiments in Lithic Technology, British Archaeologycal Reports International Series 528: 17-135. Oxford.

Rick J. (1976): Downslope Movement an Archaeological Intrasite Spatial Analysis. American Antiquity 41 (2): 133-144.

SAVELLE, J. (1984): Cultural and Natural Formation Processes of a Historic Inuit Snow Dwelling Site, Somerset Isiand, Arctic Canada. American Antiquity. 49 (39):508-524.

SCHIFFER, M. B. (1972): Archaeological Context and Systemic Context. American Antiquity $37(2): 156-165$.

- (1983): Toward the Identification of Formation Processes. American Antiquity 48 (4):675706.

- (1987): Formation Processes of the Archaeological Record. University Of New Mexico Press, Albuquerque.

Shennan, S. (1982): Exchange and Ranking: the Role of Amber in the Earlier Bronze Age of Europe. Ranking, Resource and Exchange. Aspects of the Archaeology of Early European Society. C. Renfrew y S, Shennan (Eds). 33-45.Cambridge University Press, Cambridge.

Sнотт, M. (1994): Size and Form in the Analysis of Flake Debris: Review and Recent Approaches. Journal of Archaeological Method and Theory 1(1):69-110.

- (1989): Diversity, Organization, and Behavior in the Material Record: Ethnografic and Archaeological Examples. Current Anthropology. 30(3):283-315.

SteVENSON, M. (1985): The Formation of Artifact Assemblages at Workshop/Habitation sites:models from Peace Point in Northern Alberta. American Antiquity 50 (1):63-81.

STOCKTON, E. (1973): Shaw's Creek shelter: Human Displacement of Artifacts and Its Signifance. Mankind 9: 112-117.

- (1977): Review of Early Bondaian Dates. Mankind 11: 48-51.

SUluivan, A. y K. Rozen. (1985): Debitage Analysis and Archaeological Interpretation. American Antiquity 50(4): 755-759.

TELTSER, P. (1991): Generalized Core Technology and Tool Use: A Mississippian Example. Journal of Field Archaeology 18: 363-375.

TORRENCE, R. (1989): Tools as Optimal Solutions. Time Energy and Stone Tools. R. Torrence (Ed).1-16. Cambridge University Press.

VILLA, P. (1982): Conjoinable Pieces and Site Formation Processes. American Antiquity 47: 276-290.

WHITE, P. y N. MODJESKA (1978): Dónde van Todos los Artefactos Liticos? Algunos Ejemplos y Problemas de su Distribución Social y Espacial en las Tierras Altas Papua de Nueva Guinea. La Organización Espacial de la Cultura. Hooder (Ed). 25-38. Londres, Duckwort.

YELLEN, J. (1977): Archaeological approaches to the present. Academic Press, New York. 